\title{
On the singular structure of graph hypersurfaces
}

\author{
ERIC PATTERSON
}

\begin{abstract}
I show that the singular loci of graph hypersurfaces correspond set-theoretically to their rank loci. The proof holds for all configuration hypersurfaces and depends only on linear algebra. To make the conclusion for the second graph hypersurface, I prove that the second graph polynomial is a configuration polynomial. The result indicates that there may be a fruitful interplay between the current research in graph hypersurfaces and Stratified Morse Theory.
\end{abstract}

\section{Introduction}

Let $G$ be a graph, $E$ its set of edges, and $V$ its set of vertices. For each edge $e \in E$, let $A_{e}$ be an associated variable. The first graph polynomial is

$$
\Psi_{G}(A)=\sum_{F \subset G} \prod_{e \notin F} A_{e}
$$

where the sum is over the spanning forests of $G$. A spanning forest is defined in Definition 3.3 to be a subgraph of $G$ that is a spanning tree on each component of $G$. Similarly, the second graph polynomial is

$$
\Phi_{G}(p, A)=\sum_{T \subset G} s_{T}(p) \prod_{e \notin T} A_{e},
$$

where the sum is over the quasi-spanning forests $T$ of $G, p \in K^{V, 0}$ is a momentum of $G$ and $s_{T}(p)$ is the momentum of $p$ on $T$ (see Definitions 3.4 to 3.6). In the physics and graph theory literature, these polynomials are also called Symanzik polynomials and Kirchoff polynomials. They appear in parametric Feynman integrals in quantum field theory of the form

$$
I_{G}(p)=\int_{\sigma} d A \frac{\mathrm{e}^{\mathrm{i} \frac{\Phi_{G}(p, A)}{\Psi_{G}(A)}}}{\Psi_{G}(A)^{2}},
$$

where $\sigma$ is the domain of real values of the variables $A_{e}$ that sum to 1 (see $[9,16])$. Broadhurst and Kreimer $[3,4]$ related the values of $I_{G}(p)$ to 
multiple zeta values in many cases where the momentum $p$ factors out of the integral trivially.

The results of Broadhurst and Kreimer motivated researchers to understand the correspondence between graphs and multiple zeta values via motives. For the case of where $p$ factors out of $I_{G}(p)$, Bloch, Esnault and Kreimer [2] have interpreted Equation (1.1) as the period of the mixed Hodge structure of the graph hypersurface $X_{G}$ defined by $\Psi_{G}(A)=0$. In particular, the middle dimensional cohomology of the graph hypersurface needs to be computed.

For the case of wheel-and-spokes graphs, Bloch, Esnault and Kreimer have produced such a computation in [2], and Doryn [10] has extended their techniques to zig-zag graphs. Aluffi and Marcolli [1] show that the mixed Hodge structure for three loop graphs is mixed Tate, which suggests that the period could be a multiple zeta value. Brown [8] derived the mixed Tate conclusion for a whole class of graphs for which he was also able to prove that the Feynman integrals evaluate to multiple zeta values. Brown's results provide an explanation for the known analytic computations of Feynman integrals and suggest where to look for non-multiple zeta values.

The goal of this paper is to extend the toolbox of cohomological tools available to connect Feynman integrals and multiple zeta values. To this end, I prove some simple results on the structure of the singular loci of graph hypersurfaces. I hope that these formulas may provide some tools for applying Stratified Morse Theory [11] to the study of the periods of mixed Hodge structures of graph hypersurfaces.

In particular, I am able to identify the multiplicity of the points in the graph hypersurfaces as specific rank loci. More specifically, following Proposition 2.2 in [2], the first graph polynomial is the determinant of a certain canonical bilinear form on $K^{E}$ restricted to the subspace $H_{1}(G, K)$. Here $K^{E}$ is the vector space over a field $K$ with basis $E$, and $H_{1}(G, K)$ is the first homology of the graph $G$ with coefficients in $K$. Similarly, Proposition 3.3 shows that the second graph polynomial is the determinant of the same canonical bilinear form on $K^{E}$ but restricted to a relative homology subspace $H_{1}(G, p)$ defined in Definition 3.7. Therefore, these polynomials can be analyzed with the linear algebra of configurations and configuration polynomials.

The main result, Theorem 4.1, proves that the multiplicity of a point in the hypersurface defined by a configuration polynomial is equal to its corank as a bilinear form. In particular, the theorem holds for the first and second graph hypersurfaces by Proposition 2.2 in [2] and Proposition 3.3 here. 
The approach via configuration polynomials that I present in this paper is complementary to the usual approach to graph polynomials via the graph Laplacian (see the review [9]). In particular, the graph polynomials may be derived as determinants of submatrices of the graph Laplacian, which is the content of the all-minors matrix-tree theorem. The graph Laplacian is defined in terms of the vertices of the graph, and the all-minors matrixtree theorem must verify that certain choices of submatrix do not affect the computation. For comparison, the configuration approach lifts the computation from the vertices to the edges via the homology boundary map. The choice of submatrix in the graph Laplacian approach becomes the choice of a representing element in a homology group for the configuration approach. This analogy suggests that the most general form of the all-minors matrixtree theorem may have an analogous expression in terms of configuration polynomials.

The configuration hypersurfaces naturally map to the generic symmetric determinantal varieties. Moreover, the generic symmetric determinantal varieties satisfy the same relationship between the corank and the multiplicity of their points (e.g., see Theorem 22.33 in [13]). The basic idea behind applying Stratified Morse Theory suggested by Bloch [7] is to find a stratified Morse function for the generic symmetric determinantal variety relative to its rank stratification, which could draw on a host of well-known results about the geometry of these varieties. Provided such a function, its restriction to the configuration hypersurface would be a stratified Morse function, and I hope that my result, by identifying the strata by their multiplicity, could provide some ideas for how to generate new results on the topology of the configuration hypersurface. I also note that Brown [8] uses Stratified Morse Theory following a different approach than the one just mentioned.

Among applications that I can prove, I provide a formula for the tangent cones of a graph hypersurface over an algebraically closed field of characteristic zero in Proposition 4.1. In addition, $H_{1}(G, K)$ is a subspace of $H_{1}(G, p)$, which provides some further relationships between the first and second graph hypersurfaces, for which I refer to [19]. For some applications, one may need to strengthen these results from the perspectives of both algebraic geometry and physics. The identification of multiplicity loci with degeneracy loci does not make the identification at the scheme level, and I only work in the context of a scalar field. Bloch and Kreimer show how to extend the configuration definition of the second graph polynomial from a scalar field to quaternions in [6].

I begin in Section 2 with some elementary results on bilinear forms. Section 3 recalls the definitions of configurations, configuration polynomials 
and how graph polynomials fit into these definitions. Section 4 proves the main multiplicity-corank correspondence and its consequences. I conclude in Section 5.

\section{Symmetric bilinear forms}

This section has two purposes. First, it reviews the terminology and notation on symmetric bilinear forms that I will use. Second, it proves a fundamental lemma about degenerate symmetric bilinear forms: Lemma 2.5. The results generalize to nonsymmetric bilinear forms, but there are no uses of that generality in this paper. The reader familiar with degenerate bilinear forms may prefer to skip this section. The reader seeking more details should consult [19].

\subsection{Notation and basic definitions}

I assume all vector spaces are finite dimensional. Let $V$ be a vector space over a field $K$. Denote the dual space by $V^{\vee}=\operatorname{Hom}_{K}(V, K)$. A bilinear form $B$ on $V$ is an element of $(V \otimes V)^{\vee}=V^{\vee} \otimes V^{\vee}$. A bilinear form $B$ is symmetric if $B(v \otimes w)=B(w \otimes v)$ for all $v, w \in V$.

A symmetric bilinear form $B$ defines a linear map from $V$ to $V^{\vee}$ :

$$
\begin{aligned}
0 \rightarrow \operatorname{rad}(V, B) \rightarrow V & \stackrel{\ell_{B}}{\longrightarrow} V^{\vee} \\
\ell_{B}(v) & =B(v, \cdot)=B(v \otimes \cdot) .
\end{aligned}
$$

Definition 2.1 (Radical). The radical of $B$ in $V, \operatorname{rad}(V, B)$, is

$$
\operatorname{rad}(V, B)=\operatorname{Ker} \ell_{B}=\{v \in V \mid B(v, w)=0 \text { for all } w \in V\}
$$

Definition 2.2 (Degenerate). The bilinear form $B$ is called degenerate if $\operatorname{rad}(V, B) \neq 0$.

I will often work with one symmetric bilinear form $B$ and restrict it to various subspaces $W$ of $V$. In that case, I may suppress the $B$ from the notation and write $\operatorname{rad} V$ for $\operatorname{rad}(V, B), \operatorname{rad} W$ for $\operatorname{rad}\left(W,\left.B\right|_{W}\right)$.

The rank of $B$ on $V$ is the rank of the linear map $\ell_{B}$, and the corank of $B$ on $V$ is

$$
\operatorname{corank} B=\operatorname{dim} \operatorname{rad}(V, B)=\operatorname{dim} V-\operatorname{rank} B .
$$

When I restrict $B$ to a subspace $W$, I will let $\operatorname{rank}_{W} B$ and corank $W$ denote $\operatorname{rank}\left(\left.B\right|_{W}\right)$ and corank $\left(\left.B\right|_{W}\right)$. In particular, $\operatorname{rank}_{V} B$ and $\operatorname{corank}_{V} B$ are 
synonyms for rank $B$ and corank $B$ that include $V$ in the notation. The following lemma is well-known, and I do not include the proof. I make frequent use of the equivalence of these conditions, often without comment.

Lemma 2.1. Suppose that $B$ is a symmetric bilinear form on $V$. The following conditions on $B$ are equivalent:

1. $\operatorname{corank} B>\operatorname{dim} V-k$,

2. $\operatorname{rank} B<k$ and

3. all $k \times k$ minors of $M$ vanish for a matrix $M$ representing $B$ in some basis of $V$.

A hyperplane in $V$ is a linear subspace of $V$ of codimension one (i.e., I am considering hyperplanes in the linear subspace sense, not the affine sense).

Definition 2.3. A set of hyperplanes $\left\{H_{1}, \ldots, H_{k}\right\}$ in $V$ is complete if $\cap_{i=1}^{k} H_{i}=0$. Note that $k$ may be greater than the dimension of $V$ in this definition.

Every hyperplane is the kernel of a linear form on $V$, and scaling the linear form does not change the kernel. Therefore, each hyperplane $H$ in $V$ is identified with a point $H^{\vee}$ in $\mathbb{P}\left(V^{\vee}\right)$. I mention this well-known fact so the following proposition makes sense.

Proposition 2.1 (Complete equivalent to spanning). A set of hyperplanes $\left\{H_{1}, \ldots, H_{k}\right\}$ in $V$ is complete if and only if $\left\{H_{1}^{\vee}, \ldots, H_{k}^{\vee}\right\}$ span $V^{\vee}$.

This fact is well-known, so I will not repeat the proof. Spanning is probably the preferred adjective, but I prefer the term complete to emphasize that I will use the intersection property.

\subsection{Fundamental lemmas}

The main result of this section is Lemma 2.5, which translates bounds on the rank of a degenerate symmetric bilinear form restricted to a complete set of hypersurfaces to a bound on the rank of the unrestricted bilinear form. It is the key lemma in Theorem 4.1, which identifies the multiplicities of configuration hypersurfaces with rank loci. 
Let $V$ be a vector space, and let $B$ be a symmetric bilinear form on $V$. For every subspace $W$ of $V$, the radicals of $B$ fit into the following diagram:

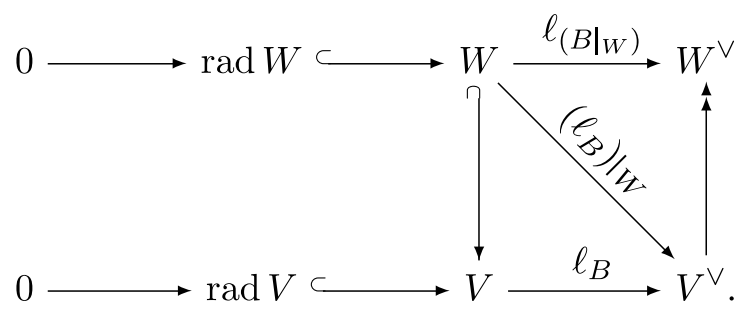

The rows are left exact by the definition of the radical. A key point to note is that there is no natural map between the radicals of $V$ and $W$.

Lemma 2.2. Let $B$ be a symmetric bilinear form on a vector space $V$, and let $W$ be a subspace of $V$. Then

$$
W \cap \operatorname{rad} V \subseteq \operatorname{rad} W
$$

Proof. Consider an element $w \in W \cap \operatorname{rad} V$. As an element of $\operatorname{rad} V$, $B(v, w)=0$ for all $v \in V$ and, in particular, for all $v \in W \subseteq V$. Because $w$ is in $W$, this proves that $w \in \operatorname{rad} W$.

Alternatively, note that $W \cap \operatorname{rad} V$ is the kernel of the diagonal map, $\left.\left(\ell_{B}\right)\right|_{W}$, in the diagram (2.1). This kernel is a subspace of $\operatorname{rad} W$, which is a consequence of the commutativity of the diagram.

Corollary 2.1. If $\operatorname{rad} V \subseteq W$, then $\operatorname{rad} V \subseteq \operatorname{rad} W$.

Let $S$ be a subset of $V$, and define the subspace orthogonal to $S$,

$$
\perp(S)=\{v \in V \mid B(v, s)=0 \text { for all } s \in S\} .
$$

For every set $S, \perp(S)$ contains $\operatorname{rad} V$. Note that $W$ is always contained in $\perp(\operatorname{rad} W)$. Therefore,

Lemma 2.3. For every subspace $W$ of $V$,

$$
W+\operatorname{rad} V \subseteq \perp(\operatorname{rad} W) .
$$

Corollary 2.2. If $W+\operatorname{rad} V=V$, then $\operatorname{rad} W \subseteq \operatorname{rad} V$. 
Proof. By Lemma 2.3,

$$
V=W+\operatorname{rad} V \subseteq \perp(\operatorname{rad} W),
$$

which implies $V=\perp(\operatorname{rad} W)$. In other words, $B(v, w)=0$ for every $v \in V$ and every $w \in \operatorname{rad} W$, which means $\operatorname{rad} W \subseteq \operatorname{rad} V$.

Corollary 2.3. If $H$ is a hyperplane in $V$, then $\operatorname{rad} V \subseteq \operatorname{rad} H$ or $\operatorname{rad} H \subseteq$ $\operatorname{rad} V$.

Proof. If $\operatorname{rad} V \subseteq H$, then $\operatorname{rad} V \subseteq \operatorname{rad} H$ by Corollary 2.1. If $\operatorname{rad} V \nsubseteq H$, then $H+\operatorname{rad} V=V$ because $H$ is a hyperplane. Then Corollary 2.2 implies that $\operatorname{rad} H \subseteq \operatorname{rad} V$.

Lemma 2.4. If $B$ is degenerate on $V$, then every complete set of hyperplanes has an element $H$ for which $\operatorname{rank}_{V} B=\operatorname{rank}_{H} B$.

Proof. If $\operatorname{rad} V$ is contained in every hyperplane in a complete set, then $\operatorname{rad} V$ is contained in their intersection, which is zero. However $B$ is degenerate, so $\operatorname{rad} V$ must be nonzero, and therefore, there is at least one hyperplane $H$ in the complete set for which $\operatorname{rad} V \nsubseteq H$. By Corollaries 2.1 and 2.3, $\operatorname{rad} H \varsubsetneqq \operatorname{rad} V$. Therefore, $\operatorname{corank}_{H} B<\operatorname{corank}_{V} B$, and thus,

$$
\begin{aligned}
n-1- & \operatorname{rank}_{H} B<n-\operatorname{rank}_{V} B \\
\operatorname{rank}_{H} B & >\operatorname{rank}_{V} B-1 \\
\operatorname{rank}_{H} B & \geq \operatorname{rank}_{V} B .
\end{aligned}
$$

The ranks are equal to the dimensions of the images of $\ell_{\left(\left.B\right|_{H}\right)}$ and $\ell_{B}$, respectively. The factorization of $\ell_{\left(\left.B\right|_{H}\right)}$ depicted in diagram (2.1) implies that

$$
\operatorname{dim} \operatorname{Im} \ell_{\left(\left.B\right|_{H}\right)} \leq \operatorname{dim} \operatorname{Im} \ell_{B}
$$

hence $\operatorname{rank}_{H} B=\operatorname{rank}_{V} B$.

Lemma 2.5 (Criterion to Bound Rank). Suppose that $B$ is a degenerate symmetric bilinear form on $V$ and $k$ is an integer, $1 \leq k<\operatorname{dim} V$. Let $\left\{H_{1}, \ldots, H_{\ell}\right\}$ be a complete set of hyperplanes in $V$. For a nonempty subset $J \subseteq\{1, \ldots, \ell\}$, define

$$
H_{J}=\cap_{j \in J} H_{j}
$$

If $\left.B\right|_{H_{J}}$ is degenerate for all subsets $J$ with $|J| \leq k$, then

$$
\operatorname{rank}_{V} B<\operatorname{dim} V-k \text {. }
$$


Proof. Proceed by induction on $\operatorname{dim} V$. For each step of the induction, I must verify the lemma for the whole set of positive integers $k$ less than $\operatorname{dim} V$. If $\operatorname{dim} V=1$, there are no positive integers $k$ less than $\operatorname{dim} V$ and no nonzero hyperplanes, so the statement is empty and there is nothing to prove.

If $\operatorname{dim} V=2$, then $k=1$ is the only positive integer less than $\operatorname{dim} V$. By Lemma 2.4 , there is a hyperplane (i.e., a line) $H_{j}$ in the complete set for which

$$
\operatorname{rank}_{V} B=\operatorname{rank}_{H_{j}} B
$$

By assumption, $\left.B\right|_{H_{j}}$ is degenerate, so $\operatorname{rank}_{H_{j}} B<\operatorname{dim} H_{j}=1$. That is, $\operatorname{rank}_{V} B=0$, which is less than $n-k=2-1=1$.

Suppose the lemma is true for vector spaces of dimension $n-1$, and suppose $\operatorname{dim} V=n$. By Lemma 2.4, there is a hyperplane in the complete set, say $H_{1}$, for which

$$
\operatorname{rank}_{V} B=\operatorname{rank}_{H_{1}} B
$$

The inductive hypothesis applies to the hyperplane $H_{1}$ and the complete set of hyperplanes in $H_{1}$ :

$$
\left\{H_{1} \cap H_{j} \mid j=2, \ldots, \ell \text { and } H_{1} \neq H_{j}\right\} .
$$

By assumption, $\left.B\right|_{H_{J}}$ is degenerate for all subsets $J \subseteq\{1, \ldots, \ell\}$ with $|J| \leq$ $k$. In particular, $\left.\left(\left.B\right|_{H_{1}}\right)\right|_{H_{\tilde{J}}}$ is degenerate for each set $\tilde{J} \subseteq\{2, \ldots, \ell\}$ with $|\tilde{J}| \leq k-1$. Then the conclusion of the inductive hypothesis is

$$
\operatorname{rank}_{V} B=\operatorname{rank}_{H_{1}} B<n-1-(k-1)=n-k .
$$

\section{Configurations}

In [2], Bloch, Esnault and Kreimer show how the first graph polynomial is a configuration polynomial. Although graph polynomials are my main interest, the language of configurations is useful when results depend only on linear algebra and not the combinatorics of graphs. In this section, I recall the language of configurations, the main formula for configuration polynomials, and how the first and second graph polynomials are specific examples of configuration polynomials. 


\subsection{Configuration polynomials}

A based vector space is a pair $(V, E)$ of a vector space $V$ and a preferred basis $E$. There is an isomorphism ${ }^{1}$ of based vector spaces between $V$ and $K^{E}$, the vector space generated by the set $E$. Every element $v$ of $V$ can be written uniquely as $\sum_{e \in E} v_{e} e$ where $v_{e} \in K$. The $v_{e}$ are the coordinates of $v$ in the basis $E$. Denote the coordinate functions

$$
\begin{aligned}
X_{e}: V & \rightarrow K \\
v & \mapsto v_{e} .
\end{aligned}
$$

Note that the whole basis $E$ is needed to define each functional $X_{e}$. Squaring the coordinate functions defines symmetric, rank-one bilinear forms on $V$ :

$$
\begin{aligned}
& X_{e}^{2}: V \otimes V \rightarrow K \\
& v \otimes w \\
& \mapsto X_{e}(v) X_{e}(w)=v_{e} w_{e}
\end{aligned}
$$

For convenience, let $X_{e}^{2}(v)=X_{e}^{2}(v, v)$ and, more generally, $b(v)=b(v, v)$ for bilinear forms $b$ on $V$. Define the natural map from $V$ to the span of these bilinear forms:

$$
\begin{gathered}
B_{E}: V \rightarrow \operatorname{Sym}^{2} V^{\vee} \\
a=\sum_{e \in E} a_{e} e \mapsto \sum_{e \in E} a_{e} X_{e}^{2} .
\end{gathered}
$$

Suppose that $E=\left\{e_{1}, \ldots, e_{n}\right\}$ so that $a=\sum_{i=1}^{n} a_{e_{i}} e_{i}$, and let the coordinates $a_{e_{i}}$ be denoted $a_{i}$. Each bilinear form $B_{E}(a)$ is diagonal when represented in the basis $E$ :

$$
B_{E}(a)=\left(\begin{array}{cccc}
a_{1} & 0 & \cdots & 0 \\
0 & a_{2} & \cdots & 0 \\
\vdots & \vdots & \ddots & \vdots \\
0 & 0 & \cdots & a_{n}
\end{array}\right)
$$

In particular, $\operatorname{det} B_{E}(a)=a_{1} a_{2} \cdots a_{n}$.

Let $\left\{A_{e}\right\}_{e \in E}$ denote the coordinate functions defined by $E$ on $V$. Note that $A_{e}=X_{e}$; the change of notation is useful for distinguishing maps from

\footnotetext{
${ }^{1} \mathrm{~A}$ morphism of based vector spaces takes basis elements to basis elements or zero.
} 
$V$ into $\operatorname{Sym}^{2} V^{\vee}$ from elements of $\operatorname{Sym}^{3} V^{\vee}$. When the elements of $E$ are enumerated $e_{1}, \ldots, e_{n}$, write $A_{i}$ for $A_{e_{i}}$. Polynomial functions on $V$ can be expressed in terms of $\left\{A_{e}\right\}_{e \in E}$, so the function $B_{E}$ can be written

$$
B_{E}=\left(\begin{array}{cccc}
A_{1} & 0 & \cdots & 0 \\
0 & A_{2} & \cdots & 0 \\
\vdots & \vdots & \ddots & \vdots \\
0 & 0 & \cdots & A_{n}
\end{array}\right)
$$

I write $B_{E}(A)$ when I want to emphasize this representation of the map $B_{E}$. The family $B_{E}(A)$ maps $V$ into $\mathrm{Sym}^{2} V^{\vee}$, and the ideal $\left\langle A_{1} A_{2} \cdots A_{n}\right\rangle$ in $K\left[A_{1}, \ldots, A_{n}\right]$ defines the locus of points in $V$ whose images in $\mathrm{Sym}^{2} V^{\vee}$ are degenerate, namely the coordinate hyperplanes for the basis $E$. The family $B_{E}(A)$ is homogeneous in the coordinates and $B_{E}(a)$ is nonzero for nonzero $a$, so $B_{E}(A)$ can also be considered as mapping $\mathbb{P}(V)$ to $\mathbb{P}\left(\operatorname{Sym}^{2} V^{\vee}\right)$.

Definition 3.1 (Configurations). A configuration $W$ is a subspace of a based vector space ${ }^{2}$.

Each configuration $W \subseteq V$ defines a family of bilinear forms $\left.B_{E}\right|_{W} \subseteq$ $\mathrm{Sym}^{2} W^{\vee}$ parametrized by $V$ by composing with the natural map:

$$
\left.B_{E}\right|_{W}: V \stackrel{B_{E}}{\longrightarrow} \mathrm{Sym}^{2} V^{\vee} \rightarrow \mathrm{Sym}^{2} W^{\vee}
$$

I write $\left.X_{e}\right|_{W}$ and $\left.B_{E}(a)\right|_{W}$ when I want to emphasize that I have restricted to $W$.

I can write $\left.B_{E}(a)\right|_{W}$ as a matrix in any basis of $W$. When I do so, the choice of basis for $W$ is often suppressed from the notation. As a matrix, $\left.B_{E}(a)\right|_{W}$ is still symmetric but is not diagonal unless $W$ is the span of a subset of $E$. If $\operatorname{dim} W=\ell$, then $\left.B_{E}(a)\right|_{W}$ is $\ell \times \ell$. As matrices with variable entries, the elements of the family $\left.B_{E}\right|_{W}$ have entries linear in $A_{1}, \ldots, A_{n}$.

\footnotetext{
${ }^{2} \mathrm{~A}$ generalized configuration is a linear map $\varphi$ from a vector space $W$ to a based vector space. In particular, for every generalized configuration $\varphi$, the image $\varphi(W)$ is a (nongeneralized) configuration in the based vector space. The results in this paper extend to generalized configurations. I do not need this generality, so I refer the interested reader to [19].
} 
Example 3.1. Let $E=\left\{e_{1}, e_{2}, e_{3}\right\}$, and let $W$ be the configuration spanned by $\ell_{1}=e_{1}+e_{2}$ and $\ell_{2}=2 e_{3}-e_{2}$. Then

$$
\begin{aligned}
X_{1}^{2}\left(\ell_{1}\right) & =1 \\
X_{2}^{2}\left(\ell_{1}\right) & =1 \\
X_{3}^{2}\left(\ell_{1}\right) & =0 \\
X_{1}^{2}\left(\ell_{2}\right) & =0 \\
X_{2}^{2}\left(\ell_{2}\right) & =1 \\
X_{3}^{2}\left(\ell_{2}\right) & =4 \\
X_{1}^{2}\left(\ell_{1}, \ell_{2}\right) & =0 \\
X_{2}^{2}\left(\ell_{1}, \ell_{2}\right) & =-1 \\
X_{3}^{2}\left(\ell_{1}, \ell_{2}\right) & =0 .
\end{aligned}
$$

Therefore, $\left.B_{E}\right|_{W}$ in the basis $\beta=\left\{\ell_{1}, \ell_{2}\right\}$ is

$$
\begin{aligned}
\left.B_{E}\right|_{W, \beta} & =A_{1}\left(\begin{array}{ll}
1 & 0 \\
0 & 0
\end{array}\right)+A_{2}\left(\begin{array}{cc}
1 & -1 \\
-1 & 1
\end{array}\right)+A_{3}\left(\begin{array}{ll}
0 & 0 \\
0 & 4
\end{array}\right) \\
& =\left(\begin{array}{cc}
A_{1}+A_{2} & -A_{2} \\
-A_{2} & A_{2}+4 A_{3}
\end{array}\right) .
\end{aligned}
$$

An element $\left.B_{E}(a)\right|_{W}$ of the family $\left.B_{E}\right|_{W}$ is degenerate if and only if

$$
\left.\operatorname{det} B_{E}(a)\right|_{W, \beta}=\left(a_{1}+a_{2}\right)\left(a_{2}+4 a_{3}\right)-a_{2}^{2}=a_{1} a_{2}+4 a_{1} a_{3}+4 a_{2} a_{3}=0 .
$$

If I write $\left.B_{E}\right|_{W}$ in a different basis $\tilde{\beta}=\left\{\tilde{\ell}_{1}, \ell_{2}\right\}$ with $\tilde{\ell}_{1}=3 \ell_{1}$, then

$$
\begin{aligned}
\left.B_{E}\right|_{W, \tilde{\beta}} & =A_{1}\left(\begin{array}{ll}
9 & 0 \\
0 & 0
\end{array}\right)+A_{2}\left(\begin{array}{cc}
9 & -3 \\
-3 & 1
\end{array}\right)+A_{3}\left(\begin{array}{ll}
0 & 0 \\
0 & 4
\end{array}\right) \\
& =\left(\begin{array}{cc}
9 A_{1}+9 A_{2} & -3 A_{2} \\
-3 A_{2} & A_{2}+4 A_{3}
\end{array}\right) .
\end{aligned}
$$

In particular, the determinant of a matrix representing $\left.B_{E}(a)\right|_{W}$ is only defined up to the squared determinant of the change of basis matrix as in

$$
\begin{aligned}
\left.\operatorname{det} B_{E}(a)\right|_{W, \tilde{\beta}} & =\left(9 a_{1}+9 a_{2}\right)\left(a_{2}+4 a_{3}\right)-9 a_{2}^{2} \\
& =9 a_{1} a_{2}+36 a_{1} a_{3}+36 a_{2} a_{3} \\
& =\left.9 \operatorname{det} B_{E}(a)\right|_{W, \beta}
\end{aligned}
$$


This relationship is well-known, and the reader desiring further clarification is referred to the references.

Definition 3.2 (Configuration polynomial). Let $W$ be a nonzero configuration in $K^{E}$. A configuration polynomial of $W, \Psi_{W}(A)$, is a generator of the principal ideal $\left.\operatorname{det} B_{E}\right|_{W}$ in $K\left[A_{1}, \ldots, A_{n}\right]$.

In particular, the determinant of $\left.B_{E}\right|_{W}$ in a basis $\beta$ for $W$ is a configuration polynomial, and I call such a configuration polynomial the configuration determinant for the basis $\beta$. Moreover, these generators are homogeneous of degree $\operatorname{dim} W$ as the determinants of $\operatorname{dim} W$ square matrices with linear entries. Now I will recall a more explicit formula for $\Psi_{W}(A)$ using Plücker coordinates.

\subsection{Configuration polynomials in terms of Plücker coordinates}

A subspace $W \subseteq V$ of dimension $\ell$ is an element of the Grassmannian of $\ell$-dimensional subspaces of $V, \operatorname{Gr}(\ell, V)$. When $V$ has a preferred basis, the configuration polynomial for $W$ can be computed using the coordinates of the Plücker embedding of $\operatorname{Gr}(\ell, V)$ into $\mathbb{P}\left(\Lambda^{\ell} V\right)$. The Plücker embedding is the map that takes an $\ell$-dimensional subspace $W$ of $V$ to the line $\operatorname{det} W=$ $\Lambda^{\ell} W$ in $\Lambda^{\ell} V$. From an ordered basis of $V$, denoted $E=\left\{e_{1}, \ldots, e_{n}\right\}$, there is an induced basis on $\bigwedge^{\ell} V$ defined by $e_{I}=e_{i_{1}} \wedge \cdots \wedge e_{i_{\ell}}$ for every set $I=$ $\left\{i_{1}, \ldots, i_{\ell}\right\}$ such that $i_{1}<\cdots<i_{\ell}$. Let $V_{I}$ denote the subspace of $V$ spanned by the $e_{i}$ for $i \in I$. By picking the basis $E$, there are projections for every $I$

$$
\begin{aligned}
\pi_{I}: V & \rightarrow V_{I} \\
\sum_{e \in E} v_{e} e & \mapsto \sum_{e \in I} v_{e} e .
\end{aligned}
$$

In particular, $\operatorname{det} V_{I}$ is the line spanned by $e_{I}$.

The image of an $\ell$-plane $W$ under the Plücker embedding has projective coordinates along each $e_{I}$, and this set of projective coordinates is the set of Plücker coordinates of $W$. Fix a basis $\beta$ of $W$. For each $I$, I write $\operatorname{Plücker}_{I, \beta}(W)$ for the coordinate of $\operatorname{det} W$ along $I$ in the basis $\beta$. That is, for an $\ell$-dimensional subspace $W$ of $V$, its coordinate $\operatorname{Plücker}_{I, \beta}(W)$ is the 


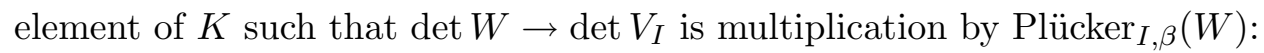

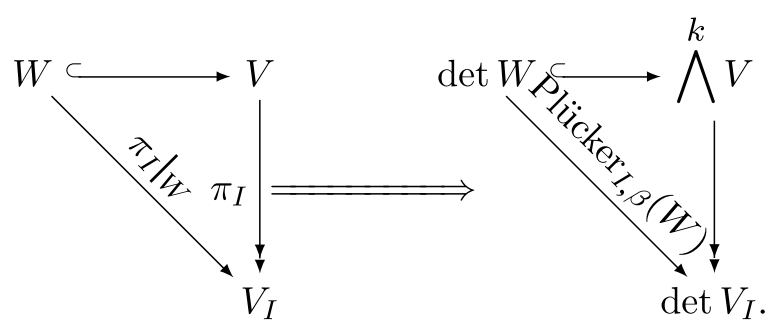

These coordinates are well-defined up to a constant representing a change of basis for $W$, and $\operatorname{Plücker}_{I, \beta}(W)$ is 0 if and only if $\left.\pi_{I}\right|_{W}: W \rightarrow V_{I}$ is not an isomorphism. A change of basis for $V$ changes the basis vectors $e_{I}$ of $\wedge^{\ell} V$, so the coordinates of $W$ change. In the context of configurations, the based vector space $K^{E}$ has a fixed preferred basis, so a general change of basis is not allowed. The preferred basis is not assumed to be ordered, and a change of basis of $K^{E}$ by reordering may change the Plücker coordinate for the index set $I$ by \pm 1 . The dependence of the coordinates on these choices of bases are suppressed from the notation.

In terms of matrices, once I have chosen the basis for $V$, I can write a basis for $W$ as $\ell$ row vectors in the coordinates of the basis for $V$. Arranging these row vectors into a $\ell \times n$ matrix, the Plücker coordinates of $W$ are the $\ell \times \ell$ minors of this matrix. The basis $E$ can be reordered to interchange columns of the $\ell \times n$ matrix representing $W$, which only changes the $\ell \times \ell$ minors by \pm 1 . Arbitrary changes of basis for $W$ changes the $\ell \times \ell$ minors by a single constant from the change of basis matrix. I formalize the preceding discussion in the next lemma.

Lemma 3.1 (Plücker coordinates are projective coordinates). If $\beta$ and $\tilde{\beta}$ are bases for $W$ and $\operatorname{Plücker}_{I, \beta}(W)$ and $\operatorname{Plücker}_{I, \tilde{\beta}}(W)$ are the respective Plücker coordinates, then there is a constant $C$, the determinant of the change of basis matrix from $\beta$ to $\tilde{\beta}$, such that

$$
\operatorname{Plücker}_{I, \beta}(W)=C \text { Plücker }_{I, \tilde{\beta}}(W)
$$

for all $\ell$-subsets $I$ of $E$, a basis for $V$. In particular, these coordinates are well-defined projective coordinates for the $\ell$-plane $W$.

The basis of $W$ used for the Plücker coordinates is a minor issue in the following, so $\operatorname{Plücker}_{I}(W)$ is written instead of $\operatorname{Plücker}_{I, \beta}(W)$. The reader may prefer to think in terms of a fixed basis, though I point out when and how results depend on the basis. 
Example 3.2. Consider the configuration $W$ of Example 3.1. In the ordered basis $E=\left\{e_{1}, e_{2}, e_{3}\right\}$ of $K^{E}$ and the basis $\left\{\ell_{1}, \ell_{2}\right\}$ of $W$, the matrix representing $W$ is

$$
\left(\begin{array}{ccc}
1 & 1 & 0 \\
0 & -1 & 2
\end{array}\right)
$$

In the induced basis of $\bigwedge^{2} K^{E}$, $\operatorname{det} W$ is

$$
\begin{aligned}
\operatorname{det} W & =\operatorname{det}\left(\begin{array}{cc}
1 & 1 \\
0 & -1
\end{array}\right) e_{1} \wedge e_{2}+\operatorname{det}\left(\begin{array}{ll}
1 & 0 \\
0 & 2
\end{array}\right) e_{1} \wedge e_{3}+\operatorname{det}\left(\begin{array}{cc}
1 & 0 \\
-1 & 2
\end{array}\right) e_{2} \wedge e_{3} \\
& =-e_{1} \wedge e_{2}+2 e_{1} \wedge e_{3}+2 e_{2} \wedge e_{3}
\end{aligned}
$$

Therefore, its Plücker coordinates are $[-1: 2: 2]$. Notice the relationship between the last line and the coefficients of the configuration polynomial

$$
\Psi_{W}(A)=A_{1} A_{2}+4 A_{1} A_{3}+4 A_{2} A_{3}
$$

The next proposition explains this relationship.

Proposition 3.1 (Configuration polynomial in Plücker coordinates). Let $W$ be a nonzero configuration in $K^{E}$. A configuration polynomial for $W$ is

$$
\Psi_{W}(A)=\sum_{\substack{F \subset E \\|F|=\operatorname{dim} W}} \text { Plücker }_{F}(W)^{2} \prod_{f \in F} A_{f} .
$$

Remark 3.1. The configuration polynomial is only defined up to a constant, so the ambiguity of which basis for $W$ is used to compute Plücker $_{F}(W)^{2}$ on the right side is irrelevant - it will still generate the principal ideal $\left.\operatorname{det} B_{E}\right|_{W}$. The basis for $K^{E}$ is fixed up to order, which can only change the Plücker coordinates by \pm 1 , and hence the coefficients Plücker $_{F}(W)^{2}$ are independent of the order of $E$. Also, note that there is some nonzero Plücker coordinate for every nonzero configuration $W$, so the configuration polynomial is never identically zero.

Proof. Pick a basis $\beta=\left\{w_{1}, \ldots, w_{\ell}\right\}$ for $W$, and work with the matrix $\left.B_{E}\right|_{W, \beta}$ representing $\left.B_{E}\right|_{W}$ in this basis. As noted above, the entries in $\left.B_{E}\right|_{W, \beta}$ are linear in the $A_{e}$, so the determinant is homogeneous of degree $\operatorname{dim} W$. The bilinear form $X_{e}^{2}$ has rank at most 1 on $W$. If it has rank 1 , then there is a basis for $W$ in which $X_{e}^{2}$ is represented by a matrix with 0 s 
everywhere except a 1 in the $(1,1)$ entry. Choosing $\beta$ to be this basis,

$$
\left.B_{E}\right|_{W, \beta}=A_{e}\left(\begin{array}{cccc}
1 & 0 & \ldots & 0 \\
0 & 0 & \ldots & 0 \\
\vdots & \vdots & \ddots & \vdots \\
0 & 0 & \ldots & 0
\end{array}\right)+\sum_{f \in E-e} A_{f} X_{f}^{2} .
$$

The variable $A_{e}$ only appears in one entry of $\left.B_{E}\right|_{W, \beta}$, so it appears with degree at most 1 in $\left.\operatorname{det} B_{E}\right|_{W, \beta}$. If $X_{e}^{2}$ has rank 0 on $W$, then

$$
\left.B_{E}\right|_{W, \beta}=A_{e} 0+\sum_{f \in E-e} A_{f} X_{f}^{2},
$$

and it is still true that $A_{e}$ appears with degree at most 1 in $\left.B_{E}\right|_{W, \beta}$. This suffices to show that the monomial terms of $\left.B_{E}\right|_{W, \beta}$ are products of $\ell$ distinct variables. Hence there are constants $c_{F}$ such that

$$
\left.\operatorname{det} B_{E}\right|_{W, \beta}=\sum_{\substack{F \subset E \\|F|=\operatorname{dim} W}} c_{F} \prod_{f \in F} A_{f} .
$$

Fix a subset $F$ and compute $c_{F}$ as follows. Evaluate both sides at $A_{e}=1$ for $e \in F$ and $A_{e}=0$ for $e \notin F$ to determine the constants. Evaluating the right side gives $c_{F}$. Evaluating $\left.\operatorname{det} B_{E}\right|_{W, \beta}$ gives the determinant of a bilinear form:

$$
\left.\operatorname{det} \sum_{f \in F} X_{f}^{2}\right|_{W}
$$

That is, $c_{F}=\left.\operatorname{det} \sum_{f \in F} X_{f}^{2}\right|_{W}$. With respect to the basis $\beta=\left\{w_{1}, \ldots, w_{\ell}\right\}$, the bilinear form $\left.X_{f}^{2}\right|_{W}$ is the matrix with $(i, j)$-th element $X_{f}\left(w_{i}\right) X_{f}\left(w_{j}\right)$. Thus,

$$
\left.\sum_{f \in F} X_{f}^{2}\right|_{W}=\left(\sum_{f \in F} X_{f}\left(w_{i}\right) X_{f}\left(w_{j}\right)\right)=\left(X_{f_{k}}\left(w_{i}\right)\right)\left(X_{f_{k}}\left(w_{j}\right)\right) .
$$

The matrix $\left(X_{f_{k}}\left(w_{j}\right)\right)$ is the $\ell \times \ell$ matrix representing the map

$$
\left.\pi_{F}\right|_{W}: W \rightarrow K^{F}
$$

projecting $W$ onto its $F$ coordinates (so $k$ is the row index, and $j$ is the column index), and $\left(X_{f_{k}}\left(w_{i}\right)\right)$ is the transpose. Taking determinants yields

$$
\left.\operatorname{det} \sum_{f \in F} X_{f}^{2}\right|_{W}=\left(\left.\operatorname{det} \pi_{F}\right|_{W}\right)^{2}=\operatorname{Plücker}_{F}(W)^{2} .
$$




\subsection{Graph polynomials}

I use the term graph to mean an undirected multigraph. The set of edges of a graph $G$ will be denoted $E(G)$, and the set of vertices $V(G)$. If $G$ is clear from the context, I abbreviate them to $E$ and $V$.

For every graph $G$, the first and second graph polynomials appear in parametric Feynman integrals $[9,16]$. Each edge $e$ in $E$ corresponds to a variable $A_{e}$. The graph polynomials are polynomials in these variables. The first graph polynomial is defined as

$$
\Psi_{G}(A)=\sum_{\substack{\text { spanningforests } \\ F \subset E}} \prod_{e \notin F} A_{e} .
$$

The definition of spanning forest differs from some references where a spanning forest is a forest containing all vertices. The definition is the necessary generalization of spanning tree to disconnected graphs.

Definition 3.3. A spanning forest is a forest whose components are spanning trees for the components of the graph.

Recall that the homology of a graph $G$ can be computed as the kernel and cokernel of a boundary map

$$
\partial: \mathbb{Z}^{E} \rightarrow \mathbb{Z}^{V}
$$

defined as follows. For each edge $e \in E$, the gluing map from $e$ to the vertices of $G$ is defined by mapping the boundary of $e$ to two elements of $V$ (not necessarily distinct). Denote these elements by $h(e)$ and $t(e)$. Fix the choice of $h(e)$ and $t(e)$ for each edge $e$. Let $\mathbb{Z}^{E}$ and $\mathbb{Z}^{V}$ be the free abelian groups on the sets $E$ and $V$. Define the group homomorphism

$$
\partial: \mathbb{Z}^{E} \rightarrow \mathbb{Z}^{V}
$$

by $\partial(e)=h(e)-t(e)$ and extend linearly. The homology of a graph $G$ is the homology of $\partial: \mathbb{Z}^{E} \rightarrow \mathbb{Z}^{V}$. That is, the first homology of $G$ is $H_{1}(G, \mathbb{Z})=$ Ker $\partial$, and the zeroth homology of $G$ is $H_{0}(G, \mathbb{Z})=$ Coker $\partial$. The definition depends on the orientation of the edges (i.e., the choice of $h(e)$ and $t(e)$ ), but the homology groups are well-defined up to isomorphism. To get a configuration, tensor with a field $K$ :

$$
0 \rightarrow H_{1}(G, \mathbb{Z}) \otimes K \rightarrow K^{E} \stackrel{\partial \otimes \mathrm{id}}{\longrightarrow} K^{V} \rightarrow H_{0}(G, \mathbb{Z}) \otimes K \rightarrow 0 .
$$


By direct calculation or the universal coefficient theorem,

$$
H_{i}(G, K)=H_{i}(G, \mathbb{Z}) \otimes K .
$$

I write $H_{i}(G)$ for $H_{i}(G, K)$ and $h_{i}(G)$ for $\operatorname{dim}_{K} H_{i}(G)$.

Proposition 3.2 ([2], Proposition 2.2). The first graph polynomial $\Psi_{G}$ is a configuration polynomial for the configuration $H_{1}(G) \subseteq K^{E}$. It can be computed as the configuration determinant in an integral basis for $H_{1}(G)$, i.e., a basis for $H_{1}(G, \mathbb{Z})$.

In this section, I prove the corresponding result, Proposition 3.3, for the second graph polynomial: it is a configuration polynomial for a relative homology group of $G$. Before I can provide the definitions, I review some terminology and notation.

Definition 3.4. A quasi-spanning forest is a spanning forest of $G$ with one edge removed. The complement of a quasi-spanning forest is a cut set. $^{3}$

I consider the graph as the pair of its set of vertices and its set of edges. Therefore, by removing an edge in the definition of quasi-spanning forest, the set of vertices remains unchanged. In the topological sense of a graph, only the interior of an edge is removed. In particular, removing an edge may leave an isolated vertex. More generally, if $F$ is a subgraph of $G$, I will use the somewhat unconventional notation that $G-F$ is the subgraph of $G$ with the edges of $F$ removed; the set of vertices $V(G-F)$ is the same as the set of vertices $V(G)$. This notation reflects the fact that I focus on the first homology of the graph, which is a subspace of the space of edges; isolated vertices will not alter this space.

From the definition, a quasi-spanning forest has one more connected component than $G$. If the spanning forests of $G$ have no edges, then $G$ has no quasi-spanning forests (e.g., if $G$ has no edges connecting distinct vertices); in the following discussion, I tacitly assume that the spanning forests of $G$ have edges.

\footnotetext{
${ }^{3}$ The terms in the literature that define quasi-spanning forest and cut set are not consistent, for example see $[5,6,16,18]$. In some cases, cut sets are called minimal cut sets to indicate that removing more edges would create more components. In other cases, cut sets are simply required to disconnect the graph, and the complement does not even need to be a forest. Similarly, quasi-spanning forests are often defined only for connected graphs, in which case they are often named two-trees or spanning two-trees. I believe the use of two in this term refers to the number of components, which will not be two in the generality of disconnected graphs.
} 
Definition 3.5. A momentum of $G$ is an element of

$K^{V, 0}=\left\{p \in K^{V} \mid \sum_{v \in V\left(G_{\alpha}\right)} p_{v}=0\right.$ for all connected components $G_{\alpha}$ of $\left.G\right\}$.

In other words, $p$ is an assignment of elements of the field $K$ to the vertices of $G$ that sum to zero over each connected component of $G$ (i.e., momentum is conserved on each component). The vertices of $G$ agree with the vertices of any of its quasi-spanning forests $F$, so $K^{V(G), 0} \subseteq K^{V(F)}$. Each component of $G \cap F$ is a spanning tree for a component of $G$ with two exceptions, and therefore, $p$ may not be a momentum for $F$. If the two components of $F$ that are not spanning trees of a component of $G$ are $T_{1}$ and $T_{2}$, then using the defining property of the momentum

$$
m_{T_{1}}(p)=\sum_{v \in T_{1}} p_{v}=-\sum_{v \in T_{2}} p_{v}=-m_{T_{2}}(p)
$$

Definition 3.6. The momentum associated to the quasi-spanning forest $F$ (or to the corresponding cut set $C=G-F$ ) is the following function from the momentum $K^{V(G), 0}$ to $K$ :

$$
s_{F}(p)=s_{C}(p)=m_{T_{1}}(p)^{2}=m_{T_{2}}(p)^{2}
$$

The second graph polynomial is

$$
\begin{aligned}
\Phi_{G}(p, A) & =\sum_{\substack{\text { quasi-spanning forests } \\
F \subset E}} s_{F}(p) \prod_{f \notin F} A_{f} \\
& =\sum_{\substack{\text { cut sets } \\
C \subset E}} s_{C}(p) \prod_{f \in C} A_{f} .
\end{aligned}
$$

Note that $\partial\left(K^{E}\right) \subseteq K^{V, 0}$ from the definition of $\partial$, and the inclusion is an equality by counting dimensions in the homology sequence. Therefore, for every $p \in K^{V, 0}$, there is a $q \in K^{E}$ such that $\partial q=p$.

Definition 3.7. For a nonzero momentum $p$, define the (first) homology of $G$ relative to $p$ to be $H_{1}(G, p)=\partial^{-1}(K p)=H_{1}(G) \oplus K q$. That is, it is the relative homology group for $K p$ considered as a chain complex concentrated in degree zero included in the chain complex for the graph $G$. 
More specifically, the kernel and homology of that inclusion of chain complexes give

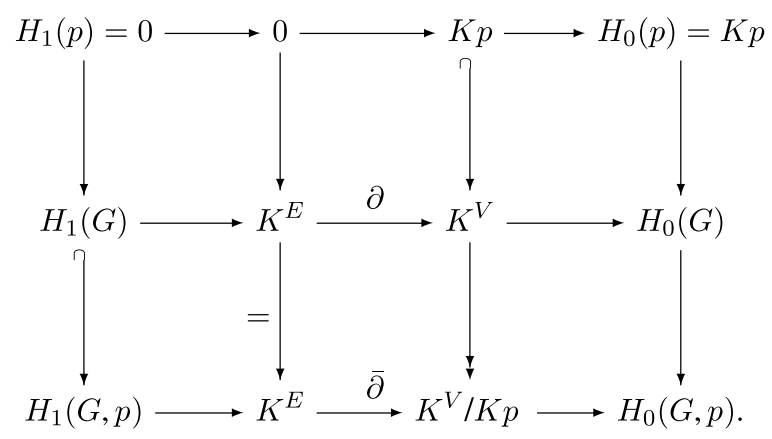

There is an induced long exact sequence from the snake lemma:

$$
0 \rightarrow H_{1}(G) \rightarrow H_{1}(G, p) \rightarrow H_{0}(p) \rightarrow H_{0}(G) \rightarrow H_{0}(G, p) \rightarrow 0
$$

Because $p \in K^{V, 0}$,

$$
H_{0}(G) \cong K^{V} / K^{V, 0} \cong K^{V} /\left(K^{V, 0}+K p\right) \cong H_{0}(G, p)
$$

and the map $H_{1}(G, p) \rightarrow H_{0}(p)=K p$ is surjective.

Proposition 3.3. The second graph polynomial $\Phi_{G}(p, A)$ is a configuration polynomial for the configuration $H_{1}(G, p) \subseteq K^{E}$. It can be computed as the configuration determinant in a basis for $H_{1}(G, p)$ consisting of an integral basis for $H_{1}(G)$ and an element $q \in K^{E}$ such that $\partial q=p$.

Remark 3.2. The definition of the homology groups depends on an arbitrarily chosen orientation of the graph. While this choice is insignificant for computing the homology group up to isomorphism, the homology group as a configuration in $K^{E}$ depends crucially on the choice. Certainly, different orientations often lead to different configurations. They all define the same configuration polynomial for the following reason.

Consider two orientations of $G$ that differ for one edge, say $e_{1}$. Suppose that $\partial$ and $\tilde{\partial}$ are the boundary maps corresponding to these two orientations. The isomorphism

$$
\begin{aligned}
\varphi: K^{E} & \rightarrow K^{E} \\
x=\left(x_{1}, \ldots, x_{n}\right) & \mapsto\left(-x_{1}, x_{2}, \ldots, x_{n}\right),
\end{aligned}
$$


satisfies $\partial \circ \varphi=\tilde{\partial}$, providing an isomorphism between the homology groups defined by the two boundary maps. Moreover, the bilinear form is invariant:

$$
B_{E}(A)(x)=\sum_{i=1}^{n} A_{i} x_{i}^{2}=B_{E}(A)(\varphi(x)) .
$$

For orientations that differ at more edges, $B_{E}(A)$ is invariant under the isomorphism defined as a composition of similarly defined $\left\{\varphi_{j}\right\}_{j \in J}$ for the set of edges $J$ at which the orientation differs.

The proof of Proposition 3.3 is an exercise in the linear algebra of based vector spaces, which I break up into a few lemmas.

Lemma 3.2 (Lifting momenta from vertices to edges). Let $F$ be a quasispanning forest of a graph $G$, let $C$ be its complementary cut set, and let $T_{1}$ and $T_{2}$ be the two maximal subtrees of $F$ that are not spanning trees of $G$. Let $p$ be a nonzero element of $K^{V, 0}$, and let $q \in K^{E}$ map to $p: \partial q=p$. Then

$$
m_{T_{i}}(p)=\sum_{\substack{e \in C \\ h(e) \in T_{i}}} q_{e}-\sum_{\substack{e \in C \\ t(e) \in T_{i}}} q_{e}
$$

for both $i=1,2$. In particular, the momentum associated to $F$ is

$$
s_{F}(p)=\left(\sum_{\substack{e \in C \\ h(e) \in T_{i}}} q_{e}-\sum_{\substack{e \in C \\ t(e) \in T_{i}}} q_{e}\right)^{2} .
$$

Proof. The proof is the same for $T_{1}$ and $T_{2}$; I prove it for $T_{1}$. The basis element $v \in K^{V}$ has a dual element $X_{v} \in\left(K^{V}\right)^{\vee}$. Denote the restriction of $X_{v}$ to $K^{V, 0}$ by $X_{v}$ as well. By definition of $q$ and $m_{T_{1}}(p)$,

$$
\begin{aligned}
m_{T_{1}}(p) & =\sum_{v \in V\left(T_{1}\right)} p_{v} \\
& =\sum_{v \in V\left(T_{1}\right)} X_{v}(p) \\
& =\sum_{v \in V\left(T_{1}\right)} X_{v}(\partial q)
\end{aligned}
$$




$$
\begin{aligned}
& =\sum_{v \in V\left(T_{1}\right)} X_{v}\left(\sum_{e \in E(G)} q_{e} h(e)-q_{e} t(e)\right) \\
& =\sum_{v \in V\left(T_{1}\right)} \sum_{e \in E(G)} q_{e} X_{v}(h(e)-t(e)) .
\end{aligned}
$$

Let $G_{\alpha}$ be the component of $G$ containing both $T_{1}$ and $T_{2}$. The edges in $T_{2}$ and $G_{\beta}$ for $\beta \neq \alpha$ have vertices disjoint from $V\left(T_{1}\right)$, so I may remove those edges from the sum:

$$
m_{T_{1}}(p)=\sum_{v \in V\left(T_{1}\right)} \sum_{e \in E\left(G_{\alpha}-T_{2}\right)} q_{e} X_{v}(h(e)-t(e)) .
$$

Note that

$$
X_{v}(h(e)-t(e))= \begin{cases}1 & \text { if } v=h(e), v \neq t(e) \\ -1 & \text { if } v=t(e), v \neq h(e) \\ 0 & \text { otherwise }\end{cases}
$$

If both $h(e)$ and $t(e)$ are in $V\left(T_{1}\right)$, then $q_{e}$ appears in the sum (3.2) with both a plus and a minus, so it cancels out. In particular, every edge $e \in T_{1}$ has both $h(e)$ and $t(e)$ in $V\left(T_{1}\right)$, so the edges in $T_{1}$ may be removed from the sum:

$$
\begin{aligned}
m_{T_{1}}(p) & =\sum_{v \in V\left(T_{1}\right)} \sum_{e \in E\left(G_{\alpha}-T_{1}-T_{2}\right)} q_{e} X_{v}(h(e)-t(e)) \\
& =\sum_{v \in V\left(T_{1}\right)} \sum_{e \in C \cap G_{\alpha}} q_{e} X_{v}(h(e)-t(e)) .
\end{aligned}
$$

Finally, $\left.X_{v}\right|_{G-G_{\alpha}}=0$ for $v \in V\left(T_{1}\right)$, so I may put the other edges of $C$ back into the formula:

$$
\begin{aligned}
m_{T_{1}}(p) & =\sum_{v \in V\left(T_{1}\right)} \sum_{e \in C} q_{e} X_{v}(h(e)-t(e)) \\
& =\sum_{e \in C} \sum_{v \in V\left(T_{1}\right)} q_{e} X_{v}(h(e)-t(e)) \\
& =\sum_{e \in C}\left(\sum_{h(e) \in V\left(T_{1}\right)} q_{e}-\sum_{t(e) \in V\left(T_{1}\right)} q_{e}\right) .
\end{aligned}
$$


Let $F$ be a subgraph of a graph $G$. The following diagram commutes and its row and column are exact:

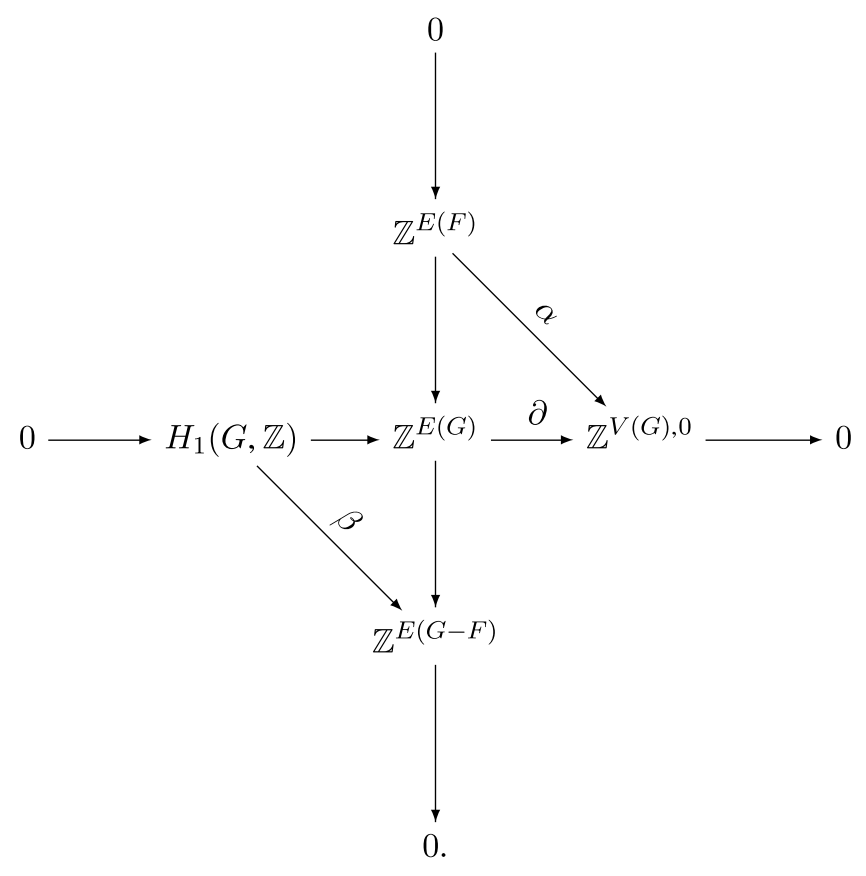

Lemma 3.3 ([2], Lemma 2.1). The subgraph $F$ is a spanning forest of $G$ if and only if $\alpha$ is an isomorphism, which is equivalent to $\beta$ being an isomorphism. In particular, $\operatorname{det}(\beta)= \pm 1$ when $F$ is a spanning forest and is zero otherwise.

Lemma 3.4 (Momenta in terms of Plücker coordinates). Let $G, F, C, T_{1}$, $T_{2}$ and $p$ be defined as in Lemma 3.2. Let $\beta$ be the projection from $H_{1}(G, p)$ to $K^{C}$. Then in a basis of $H_{1}(G, p)$ consisting of $q$ in the preimage of $p$ and an integral basis of $H_{1}(G)$,

$$
\operatorname{det}(\beta)= \pm m_{T_{1}}(p)=\mp m_{T_{2}}(p) .
$$

In particular, $\operatorname{Plücker}_{C}\left(H_{1}(G, p)\right)^{2}=s_{C}(p)$ in this basis. 
Proof. Let $m=h_{1}(G)$. Write $\beta$ in a basis as in the statement of the lemma:

$$
\left(\begin{array}{cccc}
X_{1}(q) & X_{1}\left(\ell_{2}\right) & \ldots & X_{1}\left(\ell_{m+1}\right) \\
X_{2}(q) & X_{2}\left(\ell_{2}\right) & \ldots & X_{2}\left(\ell_{m+1}\right) \\
\vdots & \vdots & \ddots & \vdots \\
X_{m+1}(q) & X_{m+1}\left(\ell_{2}\right) & \ldots & X_{m+1}\left(\ell_{m+1}\right)
\end{array}\right)
$$

The columns correspond to a $q$ such that $\partial q=p$ followed by the integral basis $\ell_{2}, \ldots, \ell_{m+1}$ of $H_{1}(G)$. The rows correspond to the elements of the cut set

$$
C=\left\{e_{1}, \ldots, e_{m+1}\right\} .
$$

Write $q_{i}$ for $X_{i}(q)$. Expand the determinant of $\beta$ along the first column:

$$
\begin{aligned}
\operatorname{det} \beta= & q_{1} \operatorname{Plücker}_{C-e_{1}}\left(H_{1}(G)\right)-q_{2} \operatorname{Plücker}_{C-e_{2}}\left(H_{1}(G)\right) \\
& +\cdots \pm q_{m+1} \operatorname{Plücker}_{C-e_{m+1}}\left(H_{1}(G)\right)
\end{aligned}
$$

The Plücker coordinate Plücker ${ }_{C-e_{i}}\left(H_{1}(G)\right)$ is computed in an integral basis, so according to Lemma 3.3,

$$
\text { Plücker }_{C-e_{i}}\left(H_{1}(G)\right)= \begin{cases} \pm 1 & \text { if }(G-C) \cup e_{i} \text { is a spanning forest } \\ 0 & \text { otherwise. }\end{cases}
$$

In particular, the coefficient of $q_{i}$ in $\operatorname{det} \beta$ vanishes if $(G-C) \cup e_{i}$ is not a spanning forest, which happens exactly when both the head and the tail of $e_{i}$ are in the same component of $F$, in agreement with Equation (3.1) where $q_{e}$ may appear twice in the sum but with opposite signs.

I must verify that the nonzero coefficients of the $q_{i}$ in Equation (3.3) agree with Equation (3.3). In this case, the subgraph $(G-C) \cup e_{i}$ is a spanning forest, the coefficients are \pm 1 , and I need to check that

1. the signs on $q_{i}$ and $q_{j}$ in $\operatorname{det} \beta$ agree if the heads of both edges $e_{i}$ and $e_{j}$ or the tails of both are in $T_{1}$;

2. and the signs on $q_{i}$ and $q_{j}$ are opposite if the head of one of the edges $e_{i}$ or $e_{j}$ and the tail of the other edge is in $T_{1}$.

Without loss of generality, assume $i=1$ and $j=2$. In either case above, $(G-C) \cup e_{1}$ is a spanning forest. At this point, the value of $\operatorname{det} \beta$ only depends on the integral basis of $H_{1}(G)$ for an overall sign, so I am free to choose the basis $\left\{\ell_{2}, \ldots, \ell_{m+1}\right\}$ of $H_{1}(G, \mathbb{Z})$. In particular, choose the basis to be a circuit basis with respect to the spanning forest $(G-C) \cup e_{1}$. Recall 
that a circuit basis with respect to a spanning forest $F$ is a basis of $H_{1}(G, \mathbb{Z})$ in which each element is a sum of edges in $F$ plus one edge not in $F$ with coefficients \pm 1 . A circuit basis exists by Lemma 3.3. For such a basis and $i, j \in\{2, \ldots, m+1\}$, the edges $e_{i}$ are not in $\ell_{j}$ unless $j=i$. For this basis, note that

$$
\begin{aligned}
& X_{i}\left(\ell_{i}\right) \neq 0 \text { for } i \in\{2, \ldots, m+1\} \\
& X_{i}\left(\ell_{j}\right)=0 \text { for } j \neq i, i, j \in\{2, \ldots, m+1\} .
\end{aligned}
$$

Then the determinant begins

$$
\operatorname{det} \beta=q_{1} \operatorname{det} \gamma_{1}-q_{2} \operatorname{det} \gamma_{2}+\cdots
$$

where

$$
\begin{array}{r}
\gamma_{1}=\left(\begin{array}{cccc}
X_{2}\left(\ell_{2}\right) & X_{2}\left(\ell_{3}\right) & \ldots & X_{2}\left(\ell_{m+1}\right) \\
X_{3}\left(\ell_{2}\right) & X_{3}\left(\ell_{3}\right) & \ldots & X_{3}\left(\ell_{m+1}\right) \\
\vdots & \vdots & \ddots & \vdots \\
X_{m+1}\left(\ell_{2}\right) & X_{m+1}\left(\ell_{3}\right) & \ldots & X_{m+1}\left(\ell_{m+1}\right)
\end{array}\right) \\
=\left(\begin{array}{cccc}
X_{2}\left(\ell_{2}\right) & X_{2}\left(\ell_{3}\right) & \ldots & X_{2}\left(\ell_{m+1}\right) \\
0 & X_{3}\left(\ell_{3}\right) & \ldots & X_{3}\left(\ell_{m+1}\right) \\
\vdots & \vdots & \ddots & \vdots \\
0 & X_{m+1}\left(\ell_{3}\right) & \ldots & X_{m+1}\left(\ell_{m+1}\right)
\end{array}\right),
\end{array}
$$

and

$$
\begin{aligned}
\gamma_{2}= & \left(\begin{array}{cccc}
X_{1}\left(\ell_{2}\right) & X_{1}\left(\ell_{3}\right) & \ldots & X_{1}\left(\ell_{m+1}\right) \\
X_{3}\left(\ell_{2}\right) & X_{3}\left(\ell_{3}\right) & \ldots & X_{3}\left(\ell_{m+1}\right) \\
\vdots & \vdots & \ddots & \vdots \\
X_{m+1}\left(\ell_{2}\right) & X_{m+1}\left(\ell_{3}\right) & \ldots & X_{m+1}\left(\ell_{m+1}\right)
\end{array}\right) \\
= & \left(\begin{array}{cccc}
X_{1}\left(\ell_{2}\right) & X_{1}\left(\ell_{3}\right) & \ldots & X_{1}\left(\ell_{m+1}\right) \\
0 & X_{3}\left(\ell_{3}\right) & \ldots & X_{3}\left(\ell_{m+1}\right) \\
\vdots & \vdots & \ddots & \vdots \\
0 & X_{m+1}\left(\ell_{3}\right) & \ldots & X_{m+1}\left(\ell_{m+1}\right)
\end{array}\right) .
\end{aligned}
$$

In fact, many other entries are zero by the choice of basis, but I have only expressed the zeros where I need them. With the exception of the first row, 
$\gamma_{1}$ and $\gamma_{2}$ agree, so by the multilinearity of the determinant,

$$
\begin{aligned}
& \operatorname{det}\left(\gamma_{1}\right) \pm \operatorname{det}\left(\gamma_{2}\right) \\
& =\operatorname{det}\left(\begin{array}{cccc}
\left(X_{2} \pm X_{1}\right)\left(\ell_{2}\right) & \left(X_{2} \pm X_{1}\right)\left(\ell_{3}\right) & \ldots & \left(X_{2} \pm X_{1}\right)\left(\ell_{m+1}\right) \\
0 & X_{3}\left(\ell_{3}\right) & \ldots & X_{3}\left(\ell_{m+1}\right) \\
\vdots & \vdots & \ddots & \vdots \\
0 & X_{m+1}\left(\ell_{3}\right) & \ldots & X_{m+1}\left(\ell_{m+1}\right)
\end{array}\right)
\end{aligned}
$$

Because I have assumed that $e_{1}$ and $e_{2}$ fit into one of the two cases above, both $(G-C) \cup e_{1}$ and $(G-C) \cup e_{2}$ are spanning forests and have no first homology. The circuit generator $\ell_{2}$ of $H_{1}\left((G-C) \cup e_{1} \cup e_{2}\right)$ must contain both $e_{1}$ and $e_{2}$ because

$$
\begin{aligned}
& h_{1}\left(\ell_{2}-e_{1}\right) \leq h_{1}\left((G-C) \cup e_{2}\right)=0, \\
& h_{1}\left(\ell_{2}-e_{2}\right) \leq h_{1}\left((G-C) \cup e_{1}\right)=0 .
\end{aligned}
$$

Therefore,

$X_{1}\left(\ell_{2}\right)=X_{2}\left(\ell_{2}\right)= \pm 1$ if only one of $e_{1}$ or $e_{2}$ has its head in $T_{1}$, and

$X_{1}\left(\ell_{2}\right)= \pm X_{2}\left(\ell_{2}\right)= \pm 1$ if both $e_{1}$ and $e_{2}$ have their heads in the same $T_{i}$.

By changing the orientation of every edge in $\ell_{2}$ if necessary, I may assume $X_{2}\left(\ell_{2}\right)=1$ so that the cases are $X_{1}\left(\ell_{2}\right)=1$ or $X_{1}\left(\ell_{2}\right)=-1$. The two cases noted above become

$$
\begin{aligned}
& \left(X_{2}-X_{1}\right)\left(\ell_{2}\right)=0 \text { if } X_{1}\left(\ell_{2}\right)=1 \\
& \left(X_{2}+X_{1}\right)\left(\ell_{2}\right)=0 \text { if } X_{1}\left(\ell_{2}\right)=-1
\end{aligned}
$$

Matching signs, the first column of the preceding matrix is zero. Therefore,

$$
\operatorname{det} \gamma_{1}= \begin{cases}\operatorname{det} \gamma_{2} & \text { if only one of } e_{1} \text { or } e_{2} \text { has its head in } T_{1} \\ -\operatorname{det} \gamma_{2} & \text { if both } e_{j} \text { have their heads in the same } T_{i}\end{cases}
$$

and

$$
\operatorname{det} \beta= \begin{cases}\left(\operatorname{det} \gamma_{1}\right)\left(q_{1}-q_{2}\right)+\ldots & \text { if only one of } h\left(e_{1}\right) \text { or } h\left(e_{2}\right) \text { is in } T_{1}, \\ \left(\operatorname{det} \gamma_{1}\right)\left(q_{1}+q_{2}\right)+\ldots & \text { if both } h\left(e_{j}\right) \in T_{i} .\end{cases}
$$

This completes the comparison of the signs on $q_{1}$ and $q_{2}$ and the proof. 
Proof of Proposition 3.3. By Proposition 3.1, the configuration determinant is

$$
\Psi_{H_{1}(G, p)}(A)=\sum_{\substack{C \subset E(G) \\|C|=h_{1}(G)+1}} \operatorname{Plücker}_{C}\left(H_{1}(G, p)\right)^{2} \prod_{e \in C} A_{e} .
$$

By Lemma 3.4, Plücker $_{C}\left(H_{1}(G, p)\right)^{2}=s_{C}(p)$ when $C$ is a cut set.

The final step is to show that if $\operatorname{Plücker}_{C}\left(H_{1}(G, p)\right)^{2}$ is nonzero, then $C$ is a cut set. There is a commutative diagram exact in the row and column

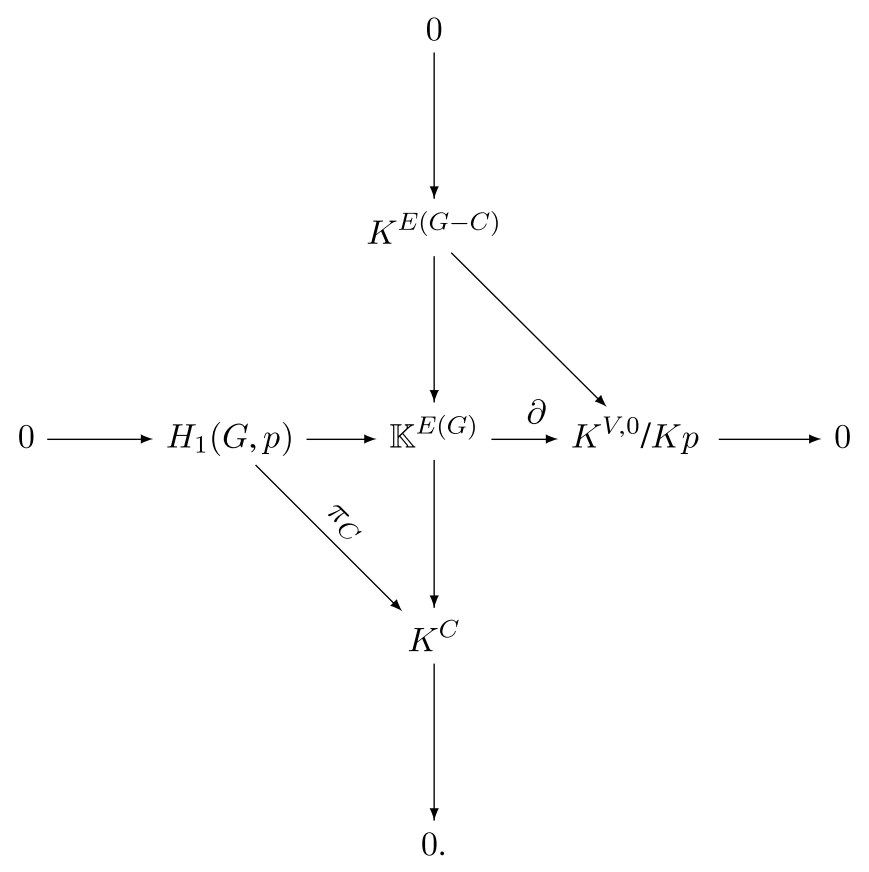

Note that Plücker ${ }_{C}\left(H_{1}(G, p)\right)$ is the determinant of $\pi_{C}$ in some basis, so if Plücker $_{C}\left(H_{1}(G, p)\right)$ is nonzero, then $\pi_{C}$ is an isomorphism. For every edge $e \in C$, the natural map $\beta_{e}: H_{1}(G) \rightarrow K^{C-e}$ factors as

$$
\beta_{e}: H_{1}(G) \hookrightarrow H_{1}(G, p) \underset{\cong}{\stackrel{\pi_{C}}{\leftrightarrows}} K^{C} \rightarrow K^{C-e} .
$$

The image of $H_{1}(G)$ in $K^{C}$ has codimension one, so there is at least one $\tilde{e} \in$ $C$ for which projecting to $K^{C-\tilde{e}}$ makes $\beta_{\tilde{e}}$ an isomorphism. By Lemma 3.3, $(G-C) \cup \tilde{e}$ is a spanning forest, which means that $C$ is a cut set.

Remark 3.3. One could define higher-order graph polynomials by replacing $K p$ with higher dimensional subspaces of $K^{V, 0}$ or even replacing $0 \rightarrow K p$ 
with other subcomplexes of $K^{E} \rightarrow K^{V, 0}$. To my knowledge, it is an open problem to prove combinatorial formulas for these generalizations of graph polynomials. Based on the review [9], I suspect such higher-order graph polynomials may correspond to the polynomials $\mathcal{W}^{(j)}$ defined there. Moreover, a general framework for such a correspondence might simply need to "lift" the all-minor matrix-tree theorem by using the differential $\partial: \mathbb{Z}^{E} \rightarrow \mathbb{Z}^{V}$.

\section{Singularities of configuration hypersurfaces}

In this section, I prove the main result, Theorem 4.1. The differentiation in this section requires me to assume that I am working over a field of characteristic zero. First, I describe how differentiating configuration polynomials yields other configuration polynomials in Section 4.1. The main result is proven in Section 4.2. An application to tangent cones is provided in Section 4.3 where I require the field to be algebraically closed.

\subsection{Restrictions of configurations}

Every based vector space $K^{E}$ has natural based subspaces $K^{F}$ for $F \subseteq E$. For a configuration $W$, there is an induced configuration in $K^{F}$ : the restriction $W^{F}=K^{F} \cap W$. Every configuration is a pair of a based vector space and a subspace, so $\left(W^{F}, K^{F}\right)$ and $\left(W^{F}, K^{E}\right)$ are two different configurations. For example, if $W \subseteq K^{F} \subseteq K^{E}$ for some subset $F \subseteq E$, then the formulas for the configuration polynomials given by Proposition 3.1 are

$$
\Psi_{\left(W, K^{F}\right)}(A)=\sum_{\substack{G \subset F \\|G|=\operatorname{dim} W}} \operatorname{Plücker}_{G}(W)^{2} \prod_{g \in G} A_{g}
$$

and

$$
\Psi_{\left(W, K^{E}\right)}(A)=\sum_{\substack{G \subset E \\|G|=\operatorname{dim} W}} \operatorname{Plücker}_{G}(W)^{2} \prod_{g \in G} A_{g}
$$

The Plücker coordinates of $\mathbb{P}\left(\bigwedge^{\ell} K^{F}\right)$ in $\mathbb{P}\left(\bigwedge^{\ell} K^{E}\right)$ vanish for all subsets $G \subset E$ that are not contained in $F$. Therefore, these polynomials are identical. I suppress the based vector space from the notation unless the context demands it. 
Example 4.1. I will continue with the configuration $W$ of Example 3.1, whose configuration polynomial I computed to be

$$
\Psi_{W}(A)=A_{1} A_{2}+4 A_{1} A_{3}+4 A_{2} A_{3} .
$$

Let $F=\left\{e_{2}, e_{3}\right\} \subseteq E$. Consider the restriction configuration $W^{F}$. In the basis $2 e_{3}-e_{2}$ for $W^{F}$, the bilinear form $B_{F}=A_{2} X_{2}^{2}+A_{3} X_{3}^{2}$ restricted from $K^{F}$ is

$$
\left.B_{F}\right|_{W_{F}}=A_{2}(1)+A_{3}(4)=\left(A_{2}+4 A_{3}\right) .
$$

The corresponding configuration polynomial is $\Psi_{W^{F}}(A)=A_{2}+4 A_{3}$. In the same basis for $W^{F}$, the bilinear form $B_{E}=A_{1} X_{1}^{2}+A_{2} X_{2}^{2}+A_{3} X_{3}^{2}$ restricted from $K^{E}$ is

$$
\left.B_{E}\right|_{W_{F}}=A_{1}(0)+A_{2}(1)+A_{3}(4)=\left(A_{2}+4 A_{3}\right) .
$$

Thus, $\Psi_{\left(W^{F}, K^{E}\right)}\left(A_{1}, A_{2}, A_{3}\right)=\Psi_{W^{F}}\left(A_{2}, A_{3}\right)$. In fact,

$$
\frac{\partial}{\partial A_{1}} \Psi_{W}\left(A_{1}, A_{2}, A_{3}\right)=\Psi_{W^{F}}\left(A_{2}, A_{3}\right) .
$$

Restrictions play a prominent role in analyzing the singularities of configuration hypersurfaces. I start with the case of $W^{E-e}$ for simplicity, and then proceed by induction. By Proposition 3.1, the configuration polynomial for a nonzero restriction $W^{E-e}$ is

$$
\Psi_{W^{E-e}}(A)=\sum_{\substack{F \subset E-e \\|F|=\operatorname{dim} W^{E-e}}} \operatorname{Plücker}_{F}\left(W^{E-e}\right)^{2} \prod_{f \in F} A_{f} .
$$

For comparison, the partial of $\Psi_{W}(A)$ with respect to $A_{e}$ is

$$
\partial_{e} \Psi_{W}(A)=\sum_{\substack{F \subset E \\|F|=\operatorname{dim} W \\ e \in F}} \operatorname{Plücker}_{F}(W)^{2} \prod_{f \in F-e} A_{f} .
$$

Here I write $\partial_{e}$ for $\partial_{A_{e}}$. Using the bijection $F \mapsto F-e$ between subsets of $E$ of size $\operatorname{dim} W$ containing $e$ and subsets $\tilde{F}$ of $E-e$ of size $\operatorname{dim} W-1$,

$$
\partial_{e} \Psi_{W}(A)=\sum_{\substack{\tilde{F} \subset E-e \\|\tilde{F}|=\operatorname{dim} W-1}} \operatorname{Plücker}_{\tilde{F} \cup e}(W)^{2} \prod_{f \in \tilde{F}} A_{f} .
$$

This last polynomial differs from $\Psi_{W^{E-e}}$ for two possible reasons: 
1. in general,

$$
\operatorname{Plücker}_{\tilde{F} \cup e}(W)^{2} \neq \operatorname{Plücker}_{\tilde{F}}\left(W^{E-e}\right)^{2} ;
$$

2. if $W \subseteq K^{E-e}$, then $W=W^{E-e}$, and the sets $\tilde{F}$ indexing the sum in $\partial_{e} \Psi_{W}(A)$ have size $\operatorname{dim} W-1$, but the sets $F$ indexing the sum in $\Psi_{W^{F}}(A)$ have size $\operatorname{dim} W$.

The following lemmas clarify these differences, which culminate in Corollary 4.1 .

Lemma 4.1 (Trivial restriction, simple case). Suppose that $W$ is a nonzero configuration in $K^{E}$. The following conditions are equivalent.

1. $W \subseteq K^{E-e}$,

2. $W=W^{E-e}$,

3. $\Psi_{W}(A)=\Psi_{W^{E-e}}(A)$,

4. $\operatorname{Plücker}_{F}(W)=\operatorname{Plücker}_{F}\left(W^{E-e}\right)$ for all $F \subseteq E$ with $|F|=\operatorname{dim} W$,

5. $\operatorname{Plücker}_{F}(W)=0$ if $e \in F,|F|=\operatorname{dim} W$,

6. $A_{e}$ does not appear in the polynomial $\Psi_{W}(A)$, and

7. $\partial_{e} \Psi_{W}(A)=0$.

Proof. The following implications are straightforward from the definitions and Proposition 3.1:

$$
(1) \Leftrightarrow(2) \Rightarrow(3) \Rightarrow(4) \Rightarrow(5) \Leftrightarrow(6) \Leftrightarrow(7) .
$$

To complete the equivalence, I show that (5) implies (1). Note that $\mathbb{P}\left(\wedge^{\ell} K^{E-e}\right)$ is the linear subspace of $\mathbb{P}\left(\wedge^{\ell} K^{E}\right)$ defined in the Plücker coordinates by Plücker $_{F}=0$ for those $F$ containing $e$. In particular, if $\operatorname{Plücker}_{F}(W)=0$ for all $F$ containing $e$, then $\operatorname{det} W \in \mathbb{P}\left(\bigwedge^{\ell} K^{E-e}\right)$. Therefore, $W \subseteq K^{E-e}$.

The previous lemma generalizes by induction to subsets of $E$ with more than one element.

Lemma 4.2 (Trivial restriction). Let $W$ be a nonzero configuration in $K^{E}$. The following are equivalent for a subset $H \subseteq E$ :

1. $W \subseteq K^{H}$, 
2. $W=W^{H}$,

3. $\Psi_{W}(A)=\Psi_{W^{H}}(A)$,

4. $\operatorname{Plücker}_{F}(W)=\operatorname{Plücker}_{F}\left(W^{H}\right)$ for all $F \subseteq E$ with $|F|=\operatorname{dim} W$,

5. $\operatorname{Plücker}_{F}(W)=0$ if $F \cap(E-H) \neq \emptyset,|F|=\operatorname{dim} W$,

6. $A_{e}$ does not appear in $\Psi_{W}(A)$ for all $e \in E-H$, and

7. $\partial_{e} \Psi_{W}(A)=0$ for all $e \in E-H$.

Proof. This follows by induction on the size of $E-H$ from Lemma 4.1.

The case when $W$ is not contained in $K^{H}$ is described by formally differentiating $\Psi_{W}(A)$. Again, I start with $H=E-e$ and proceed by induction. First, I state a well-known fact from linear algebra that I use several times:

Lemma 4.3. Suppose $U_{1}$ and $U_{2}$ are subspaces of a vector space $V$. Then

$$
\operatorname{dim} U_{1} \cap U_{2} \geq \operatorname{dim} U_{1}-\operatorname{codim} U_{2},
$$

which can be summarized as the dimension of a subspace may drop by at most $k$ when intersected with a codimension $k$ subspace.

Lemma 4.4 (Nontrivial restriction, simple case). Suppose $W$ is a configuration in $K^{E}$, and let $e$ be an element of $E$ for which $W^{E-e}$ is nonzero. The following conditions are equivalent:

1. $W \nsubseteq K^{E-e}$,

2. $W^{E-e}$ is a hyperplane in $W$, and there is a nonzero constant $C$ such that for all $F \subset E-e$ with $|F|=\operatorname{dim} W^{E-e}$

$$
\operatorname{Plücker}_{F}\left(W^{E-e}\right)=C \operatorname{Plücker}_{F \cup e}(W),
$$

3. $\left\langle\partial_{e} \Psi_{W}(A)\right\rangle=\left\langle\Psi_{W^{E-e}}(A)\right\rangle$ as ideals in the polynomial ring $K[A]$.

Proof. $(1) \Rightarrow(2)$ : By Lemma 4.3 and the assumption that $W \nsubseteq K^{E-e}$, the dimension of $W$ drops by 1 when intersected with the hyperplane $K^{E-e}$.

The projections

$$
\left.\pi_{F}\right|_{W^{E-e}}: W^{E-e} \rightarrow K^{F}
$$


have factorizations

$$
\left.\pi_{F}\right|_{W^{E-e}}: W^{E-e} \hookrightarrow W \stackrel{\left.\pi_{F \cup e}\right|_{W}}{\longrightarrow} K^{F \cup e} \rightarrow K^{F}
$$

Extend a basis $\left\{w_{1}, \ldots, w_{m-1}\right\}$ of $W^{E-e}$ to a basis for $W$ by adding an element $w_{m}$. The matrix representing the map from $W$ to $K^{F \cup e}$ in this basis for $W$ and the canonical basis of $K^{F \cup e}$ is

$$
\begin{gathered}
\left(\begin{array}{cccc}
X_{f_{1}}\left(w_{1}\right) & X_{f_{1}}\left(w_{2}\right) & \ldots & X_{f_{1}}\left(w_{m}\right) \\
X_{f_{2}}\left(w_{1}\right) & X_{f_{2}}\left(w_{2}\right) & \ldots & X_{f_{2}}\left(w_{m}\right) \\
\vdots & \vdots & \ddots & \vdots \\
X_{e}\left(w_{1}\right) & X_{e}\left(w_{2}\right) & \ldots & X_{e}\left(w_{m}\right)
\end{array}\right) \\
=\left(\begin{array}{cccc}
X_{f_{1}}\left(w_{1}\right) & X_{f_{1}}\left(w_{2}\right) & \ldots & X_{f_{1}}\left(w_{m}\right) \\
X_{f_{2}}\left(w_{1}\right) & X_{f_{2}}\left(w_{2}\right) & \ldots & X_{f_{2}}\left(w_{m}\right) \\
\vdots & \vdots & \ddots & \vdots \\
0 & 0 & \ldots & X_{e}\left(w_{m}\right)
\end{array}\right) .
\end{gathered}
$$

Here $f_{1}, \ldots, f_{m-1}$ denote the elements of $F$. Expanding the above determinant in the last row, whose only nonzero element is $X_{e}\left(w_{m}\right)$, gives

$$
\begin{aligned}
\operatorname{Plücker}_{F}(W) & =(-1)^{2 m} X_{e}\left(w_{m}\right) \operatorname{Plücker}_{F}\left(W^{E-e}\right) \\
& =X_{e}\left(w_{m}\right) \operatorname{Plücker}_{F}\left(W^{E-e}\right)
\end{aligned}
$$

In particular, $C=X_{e}\left(w_{m}\right)$ satisfies the conclusion of (2). The choice of $w_{m}$ can change the overall constant, but it is independent of the subsets $F$.

$(2) \Rightarrow(3)$ : As noted in Equation (4.1),

$$
\partial_{e} \Psi_{W}(A)=\sum_{\substack{\tilde{F} \subset E-e \\|\tilde{F}|=\operatorname{dim} W-1}} \operatorname{Plücker}_{\tilde{F} \cup e}(W)^{2} \prod_{f \in \tilde{F}} A_{f} .
$$

By the assumption (2), the coefficients can be simplified to

$$
\partial_{e} \Psi_{W}(A)=C^{2} \sum_{\substack{\tilde{F} \subset E-e \\|\tilde{F}|=\operatorname{dim} W-1}} \operatorname{Plücker}_{\tilde{F}}\left(W^{E-e}\right)^{2} \prod_{f \in \tilde{F}} A_{f} .
$$

That is,

$$
\partial_{e} \Psi_{W}(A)=C^{2} \Psi_{W^{E-e}}(A) .
$$

The ambiguity of the overall constant is removed by considering the ideals. 
$(3) \Rightarrow(1)$ : By assumption, there is a unit $C$ such that $\partial_{e} \Psi_{W}(A)=$ $C \Psi_{W^{E-e}}(A)$. By the assumption that $W^{E-e}$ is nonzero, one of its Plücker coordinates is nonzero, so $\partial_{e} \Psi_{W}(A) \neq 0$ by Proposition 3.1. If $W$ were contained in $K^{E-e}$, then $\partial_{e} \Psi_{W}(A)=0$ by Lemma 4.1, which would be a contradiction. Thus, $W \nsubseteq K^{E-e}$.

Again, I generalize to larger subsets of $E$ and higher derivatives. For $F=\left\{e_{1}, \ldots, e_{k}\right\} \subseteq E$, let $\partial_{F}$ denote $\partial_{e_{1}} \cdots \partial_{e_{k}}$.

Lemma 4.5 (Nontrivial restriction). Let $W$ be a configuration in $K^{E}$. The following conditions are equivalent for a subset $H \varsubsetneqq E$ assuming $W^{H}$ is nonzero:

1. for all sets $H^{\prime}$ with $H \varsubsetneqq H^{\prime} \subseteq E$, there exists $e \in H^{\prime}-H$ such that

$$
W^{H^{\prime}} \nsubseteq K^{H^{\prime}-e},
$$

2. $W^{H}$ is a codimension $|E-H|$ subspace of $W$, and there is a nonzero constant $C$ such that for all $F \subset H$ with $|F|=\operatorname{dim} W^{H}$,

$$
\operatorname{Plücker}_{F}\left(W^{H}\right)=C \operatorname{Plücker}_{F \cup(E-H)}(W),
$$

3. $\left\langle\partial_{(E-H)} \Psi_{W}(A)\right\rangle=\left\langle\Psi_{W^{H}}(A)\right\rangle$.

If these conditions are not satisfied for $H$, then $\Psi_{W^{H}}(A)=\Psi_{W^{H^{\prime}}}(A)$ for each $H^{\prime}$ for which condition (1) fails and $\partial_{(E-H)} \Psi_{W}(A)=0$.

Proof. The proof proceeds by induction on the size of $|E-H|$. At each stage of the induction, first prove that if $H$ does not satisfy (1), then $\Psi_{W^{H}}(A)=$ $\Psi_{W^{H^{\prime}}}(A)$ for each $H^{\prime}$ for which condition (1) fails and

$$
\partial_{(E-H)} \Psi_{W}(A)=0 .
$$

Then proceed to prove that

$$
(1) \Rightarrow(2) \Rightarrow(3) \Rightarrow(1)
$$

The reason for this complicated induction is two-fold. First, the inductive hypothesis for the equivalence of the three conditions is used to prove what happens when (1) fails. Second, the situation in which (1) fails is used to prove $(3) \Rightarrow(1)$ by contradiction.

First consider the case $|E-H|=1$. If $H$ does not satisfy (1), then Lemma 4.1 implies that $\Psi_{W^{H}}(A)=\Psi_{W}(A)$ and $\partial_{(E-H)} \Psi_{W}(A)=0$. The 
equivalence of the three conditions is the content of Lemma 4.4. Now consider $H \subseteq E$ with $|E-H|>1$.

If $H$ does not satisfy (1), there are sets $H^{\prime}$ such that

$$
H \varsubsetneqq H^{\prime} \subseteq E
$$

and for all elements $e \in H^{\prime}-H$,

$$
W^{H^{\prime}} \subseteq K^{H^{\prime}-e} .
$$

In particular, $W^{H^{\prime}} \subseteq K^{H}$ for such subsets, and thus $W^{H}=W^{H^{\prime}}$ by Lemma 4.2 .

Let $\tilde{H}$ be a maximal such subset. If $\tilde{H}=E$, then $\partial_{E-H} \Psi_{W}=0$ by Lemma 4.2. If $\tilde{H} \varsubsetneqq E$, then by maximality, for every set $H^{\prime}$ such that $\tilde{H} \varsubsetneqq H^{\prime} \subseteq E$, there is an element $e^{\prime} \in H^{\prime}-H$ such that $W^{H^{\prime}} \nsubseteq K^{H^{\prime}-e^{\prime}}$. There must be such an element $e^{\prime}$ in the smaller set $H^{\prime}-\tilde{H}$, otherwise Lemma 4.2 implies that $W^{H^{\prime}}=W^{\tilde{H}}$, and thus

$$
W^{H^{\prime}}=W^{\tilde{H}}=W^{H} \subseteq K^{H^{\prime}-e^{\prime}} .
$$

Therefore, the inductive hypothesis for the equivalence of the three conditions applies to $\tilde{H}$, so there is a nonzero constant $C$ such that

$$
\partial_{(E-\tilde{H})} \Psi_{W}(A)=C \Psi_{W^{\tilde{H}}}(A) .
$$

For every $e \in \tilde{H}-H$,

$$
\partial_{e} \Psi_{W^{\tilde{H}}}(A)=0,
$$

by Lemma 4.2. Thus,

$$
\begin{aligned}
\partial_{(E-H)} \Psi_{W}(A) & =\partial_{(\tilde{H}-H)} \partial_{(E-\tilde{H})} \Psi_{W}(A) \\
& =C \partial_{(\tilde{H}-H)} \Psi_{W \tilde{H}}(A) \\
& =0 .
\end{aligned}
$$

$(1) \Rightarrow(2)$ : Assuming the condition in (1) holds for $H$, taking $H^{\prime}=E$, there is an element $\tilde{e} \in E-H$ such that $W \nsubseteq K^{E-\tilde{e}}$. By Lemma $4.4, W^{E-\tilde{e}}$ is a codimension 1 subspace of $W$ and there is a nonzero constant $\tilde{C}$ such that

$$
\operatorname{Plücker}_{\tilde{F}}\left(W^{E-\tilde{e}}\right)=\tilde{C} \operatorname{Plücker}_{\tilde{F} \cup \tilde{e}}(W),
$$

for all $\tilde{F} \subset E-\tilde{e}$ with $|\tilde{F}|=\operatorname{dim} W^{E-\tilde{e}}$. 
The condition (1) applies to all $H^{\prime}$ with $H \varsubsetneqq H^{\prime} \subseteq E-\tilde{e}$, and

$$
|E-\tilde{e}-H|<|E-H|
$$

By the inductive hypothesis applied to the configuration $W^{E-\tilde{e}}$ in $K^{E-\tilde{e}}$, $W^{H}$ is a codimension $|E-\tilde{e}-H|$ subspace of $W^{E-\tilde{e}-H}$ and there is a nonzero constant $C^{\prime}$ such that

$$
\operatorname{Plücker}_{F}\left(W^{H}\right)=C^{\prime} \operatorname{Plücker}_{F \cup(E-\tilde{e}-H)}\left(W^{E-\tilde{e}}\right)
$$

for all $F \subset H$ with $|F|=\operatorname{dim} W^{H}$. Therefore, $W^{H}$ is a codimension $|E-H|$ subspace of $W$, and there is a nonzero constant $C=C^{\prime} \tilde{C}$ such that

$$
\begin{aligned}
\operatorname{Plücker}_{F}\left(W^{H}\right) & =C^{\prime} \text { Plücker }_{F \cup(E-\tilde{e}-H)}\left(W^{E-\tilde{e}}\right) \\
& =C^{\prime} \tilde{C} \operatorname{Plücker}_{F \cup(E-H)}(W)
\end{aligned}
$$

for all $F \subset H$ with $|F|=\operatorname{dim} W^{H}$.

$(2) \Rightarrow(3)$ : In general, using the bijection between subsets of $E$ containing $E-H$ of size $\operatorname{dim} W$ and subsets of $H$ of size $\operatorname{dim} W-|E-H|$,

$$
\begin{aligned}
\partial_{(E-H)} \Psi_{W}(A)= & \sum_{\substack{F \subseteq E \\
|F|=\operatorname{dim} W \\
E-H \subseteq F}} \operatorname{Plücker}_{F}(W)^{2} \prod_{f \in F-(E-H)} A_{f} \\
= & \sum_{\substack{\tilde{F} \subseteq H \\
|\tilde{F}|=\operatorname{dim} W-|E-H|}} \operatorname{Plücker}_{\tilde{F} \cup(E-H)}(W)^{2} \prod_{f \in \tilde{F}} A_{f} .
\end{aligned}
$$

By assumption (2), the Plücker coordinates and $\operatorname{dim} W-|E-H|$ become

$$
\begin{aligned}
\partial_{(E-H)} \Psi_{W}(A) & =C^{2} \sum_{\substack{\tilde{F} \subseteq H \\
|\tilde{F}|=\operatorname{dim} W^{H}}} \operatorname{Plücker}_{\tilde{F}}\left(W^{H}\right)^{2} \prod_{f \in \tilde{F}} A_{f} \\
& =C^{2} \Psi_{W^{H}}(A) .
\end{aligned}
$$

Therefore, the ideals are the same.

$(3) \Rightarrow(1)$ : By assumption, $W^{H}$ is nonzero, so one of its Plücker coordinates is nonzero, and Proposition 3.1 shows that $\Psi_{W^{H}}(A)$ is not identically zero. 
I prove by contradiction, so I will assume (1) fails and show that this implies that (3) fails. In fact, when (1) fails, I have already shown that

$$
\partial_{(E-H)} \Psi_{W}(A)=0
$$

which contradicts condition (3) that there is a unit $C$ such that

$$
\partial_{(E-H)} \Psi_{W}(A)=C \Psi_{W^{H}}(A) \neq 0
$$

Remark 4.1. When Lemmas 4.4 and 4.5 are applied to graph polynomials, there is no arbitrary scaling of the configuration polynomials because an integral basis fixes the coefficients to be 1 or 0 . In particular, the arbitrary nonzero constants $C$ may be replaced by 1 , and the equality of ideals may be replaced by equality of polynomials.

Moreover, if $G_{1}$ is a subgraph of $G$, then a boundary map for $G$ restricts to a boundary map for $G_{1}$. Therefore, $H_{1}\left(G_{1}\right)$ is the restriction of $H_{1}(G)$ to $K^{E\left(G_{1}\right)}$. Whether $\Psi_{G_{1}}(A)$ can be computed by $\partial_{E\left(G-G_{1}\right)} \Psi_{G}(A)$ depends on whether $E\left(G-G_{1}\right)$ is contained in the complement of a spanning forest of $G$.

Similarly for the second graph polynomial, $H_{1}\left(G_{1}, p\right)$ is the restriction of $H_{1}(G, p)$ to $K^{E\left(G_{1}\right)}$ if $p$ is a momentum on $G_{1}$. If $p$ is not a momentum on $G_{1}$, then the restriction of $H_{1}(G, p)$ to $K^{E\left(G_{1}\right)}$ is $H_{1}\left(G_{1}\right)$. Whether the restriction can be computed by differentiating depends on whether $E(G-$ $\left.G_{1}\right)$ is contained in a cut set.

The following corollary summarizes the preceding results for the applications to follow.

Corollary 4.1 (Restrictions of configuration polynomials). Let $W$ be a nonzero configuration of dimension $m$ in $K^{E}$. For every integer $k, 1 \leq k \leq$ $m-1$, the following ideals in $K[A]$ are the same

$$
\left\langle\partial_{(E-H)} \Psi_{W}(A)|| E-H \mid \leq k\right\rangle=\left\langle\Psi_{W^{H}}(A)|| E-H \mid \leq k\right\rangle .
$$

Proof. First note that the condition $k \leq m-1$ implies that for $|E-H| \leq$ $k$ :

(Lemma 4.3)

$$
\begin{aligned}
\operatorname{dim} W^{H} & =\operatorname{dim} W \cap K^{H} \\
& \geq m-k \\
& \geq m-(m-1) \\
& =1 .
\end{aligned}
$$


In particular, the configurations $W^{H}$ are nonzero, which is required for citing Lemma 4.4 below.

Proceed by induction. Let

$$
\mathcal{A}_{k}=\left\langle\Psi_{W^{H}}(A)|| E-H \mid \leq k\right\rangle
$$

and

$$
\mathcal{B}_{k}=\left\langle\partial_{(E-H)} \Psi_{W}(A)|| E-H \mid \leq k\right\rangle .
$$

Consider first the case $k=1$, and suppose $H$ is a subset for which $|E-H|=$ 1. If condition (1) of Lemma 4.4 fails for $H$, then

$$
\partial_{(E-H)} \Psi_{W}(A)=0 \in \mathcal{A}_{1}
$$

and

$$
\Psi_{W^{H}}(A)=\Psi_{W}(A) .
$$

Note that $\Psi_{W}(A)$ is an element of $\mathcal{B}_{1}$ by Euler's formula for homogeneous polynomials or by defining $\partial_{\emptyset} \Psi_{W}(A)=\Psi_{W}(A)$. If condition (1) is satisfied by $H$, then Lemma 4.4 shows that

$$
\left\langle\partial_{(E-H)} \Psi_{W}(A)\right\rangle=\left\langle\Psi_{W^{H}}(A)\right\rangle .
$$

Summing these ideals over all $H$ for which $|E-H| \leq 1$ gives $\mathcal{A}_{1}=\mathcal{B}_{1}$.

Suppose $\mathcal{A}_{k-1}=\mathcal{B}_{k-1}$, and consider an $H$ for which $|E-H|=k$. If $H$ satisfies condition (1) of Lemma 4.5, then $\left\langle\partial_{(E-H)} \Psi_{W}(A)\right\rangle=\left\langle\Psi_{W^{H}}(A)\right\rangle$ by that lemma. If $H$ does not satisfy condition (1) of Lemma 4.5, then

$$
\partial_{(E-H)} \Psi_{W}(A)=0 \in \mathcal{A}_{k}
$$

and there is an $H^{\prime} \supseteq H$ for which

$$
\Psi_{W^{H}}(A)=\Psi_{W^{H^{\prime}}}(A) \in \mathcal{B}_{k-1} \subseteq \mathcal{B}_{k} .
$$

Therefore, adding these generators to $\mathcal{A}_{k-1}$ and $\mathcal{B}_{k-1}$ for all $H$ for which $|E-H|=k$ proves $\mathcal{A}_{k}=\mathcal{B}_{k}$.

Remark 4.2. If $k \geq m$ in Corollary 4.1, then the ideal

$$
\mathcal{A}_{k}=\left\langle\Psi_{W^{H}}(A)|| E-H \mid \leq k\right\rangle
$$

may not be defined because $W^{H}$ may be zero and there is no convention for the configuration polynomial of the zero configuration. Note, however, 
that there may be $H \subseteq E,|E-H| \geq m$ for which $W^{H}$ is nonzero. For example, if $W=H_{1}(G)$ is a graph configuration, then the restriction $W^{H}$ is the configuration for the graph with edges $H$ and the same vertices [2]. If $|E-H| \geq m$, the graph with edges $H$ will have nonzero first homology as long as $H$ is not a forest.

For $k<m$, the ideals form an ascending chain

$$
\mathcal{A}_{1} \subseteq \cdots \subseteq \mathcal{A}_{m-1}
$$

If the convention for the zero configuration were that its configuration polynomial was a unit, then $\mathcal{A}_{m}=K[A]$, which completes the chain. On the other hand, the ideals

$$
\mathcal{B}_{k}=\left\langle\partial_{(E-H)} \Psi_{W}(A)|| E-H \mid \leq k\right\rangle,
$$

are defined for all $k$. In fact, $\mathcal{B}_{k}=K[A]$ for $k \geq m$ because $\partial_{(E-H)} \Psi_{W}(A)=$ $\operatorname{Plücker}_{(E-H)}(W)^{2}$ if $|E-H|=m$ by Proposition 3.1, and at least one of these Plücker coordinates is nonzero.

\subsection{Singularity-rank correspondence}

This section describes the singularities of the configuration hypersurfaces in terms of their rank. For every homogeneous ideal $I$ in $K\left[A_{1}, \ldots, A_{n}\right]$, define the variety of $I$

$$
\mathcal{V}(I)=\left\{\left[a_{1}: \cdots: a_{n}\right] \in \mathbb{P}\left(K^{E}\right) \mid f(a)=0 \text { for all homogeneous } f \in I\right\} .
$$

Often the term variety is reserved for certain types of $I$ or possibly certain types of fields $K$, but I impose no additional conditions for my usage here.

The configuration hypersurface of a configuration $W$ is $\mathcal{V}\left(\Psi_{W}\right)$. If the dimension of $W$ is $m$, then the configuration hypersurface $X_{W}$ is the degeneracy locus $D_{m-1}\left(\left.B_{E}\right|_{W}\right) \subset \mathbb{P}\left(K^{E}\right)$. Recall that a degeneracy locus of a family of matrices $M(A)$ in $\mathbb{P}\left(K^{E}\right)$ is the set of points

$$
D_{k}(M)=\left\{a \in \mathbb{P}\left(K^{E}\right) \mid \operatorname{rank} M(a) \leq k\right\} .
$$

There is a chain of degeneracy loci

$$
\begin{aligned}
D_{0}\left(\left.B_{E}\right|_{W}\right) & \subseteq D_{1}\left(\left.B_{E}\right|_{W}\right) \subseteq \cdots \subseteq \\
& \subseteq D_{m-2}\left(\left.B_{E}\right|_{W}\right) \subseteq D_{m-1}\left(\left.B_{E}\right|_{W}\right) \subseteq D_{m}\left(\left.B_{E}\right|_{W}\right)=\mathbb{P}\left(K^{E}\right)
\end{aligned}
$$


The central goal of the section, Theorem 4.1, identifies $D_{m-k-1}\left(B_{E} \mid W\right)$ for $1 \leq k \leq m-1$ as $\mathcal{V}\left(I_{k}\right)$ where

$$
I_{k}=\left\langle\partial_{F} \Psi_{W}|| F \mid \leq k\right\rangle
$$

My tools are Lemma 2.5 and the description of $I_{k}$ from Corollary 4.1. I do not use or study the ideal associated to a subset $V$ of $\mathbb{P}\left(K^{E}\right)$

$\mathcal{I}(V)=\left\langle f \in K\left[A_{1}, \ldots, A_{n}\right]\right| f$ is homogeneous and $f(a)=0$ for all $\left.a \in V\right\rangle$.

Therefore, I avoid many algebraic geometry issues that arise over nonalgebraically closed fields. I do not attempt to identify $\mathcal{I}\left(D_{k}\left(B_{E} \mid W\right)\right)$ with $I_{k}$, and I do not know whether $I_{k}$ provides a reduced scheme structure.

Definition 4.1 (Order of singularity ideal). Let $X$ be a projective variety defined by a homogeneous ideal $I$ in $K\left[A_{1}, \ldots, A_{n}\right]$. The first order singularity ideal, $S(I)$ or $S(X)$, is the ideal generated by the set

$$
\left\{\frac{\partial f}{\partial A_{i}} \mid f \in I \text { and } i=1, \ldots, n\right\}
$$

The $k$-th order singularity ideal is $S^{(k)}(X)=S^{(k)}(I)=S\left(S^{(k-1)}(I)\right)$. The locus of order at least $k$ singularities of $X, \operatorname{Sing}_{\geq k} X$, is the scheme defined by $S^{(k)}(I)$.

Note that when $X$ is a hypersurface with $I=\langle f\rangle$, the first singularity ideal $S(X)$ defines the singular locus $\operatorname{Sing} X$ because the matrix of derivatives of $f$ is zero if and only if its rank is zero. However, Sing $X$ will not be a hypersurface, so generally,

$$
\mathcal{V}\left(S^{(2)}(I)\right) \varsubsetneqq \operatorname{Sing}(\operatorname{Sing} X)
$$

In terms of the Jacobian matrix for $\operatorname{Sing} X, \operatorname{Sing}(\operatorname{Sing} X)$ is defined by a rank condition, but $\mathcal{V}\left(S^{(2)}(I)\right)$ corresponds to the Jacobian matrix being identically 0 . Rather than defining singular loci of singular loci, the $k$-th order singularity ideals filter $X$ by the degree of the tangent cones. More details on this interpretation are provided in Section 4.3. 
Euler's formula for homogeneous polynomials states that

$$
\sum_{i=1}^{n} A_{i} \frac{\partial f}{\partial A_{i}}=\operatorname{deg}(f) f
$$

Therefore, each homogeneous $f$ in $I$ is also in $S(I)$ using the assumption that the characteristic of $K$ is zero so that $\operatorname{deg}(f)$ is invertible. The ideal $I$ is homogeneous, so every $g \in I$ is also in $S(I)$ by applying the same reasoning to its homogeneous pieces. Therefore, the order of singularity ideals form a chain

$$
I \subseteq S(I) \subseteq S^{(2)}(I) \subseteq \ldots
$$

and so do the order at least $k$ singular loci defined by these ideals

$$
X \supseteq \operatorname{Sing}_{\geq 1} X \supseteq \operatorname{Sing}_{\geq 2} X \supseteq \ldots
$$

In particular, note that the $k$-th order singularity ideal can be defined by

$$
\left.S^{(k)}(I)=\left\langle\frac{\partial^{m} f}{\partial A_{1}^{j_{1}} \cdots \partial A_{n}^{j_{n}}}\right| f \in I, m \leq k, \text { and } \sum_{i=1}^{n} j_{i}=m\right\rangle .
$$

Define the points of multiplicity $k$

$$
\text { Mult }_{k} X=\operatorname{Sing}_{\geq k-1} X-\operatorname{Sing}_{\geq k} X
$$

In other words, Mult $_{k} X$ is the set of points in $X$ whose tangent cones have degree $k$.

Lemma 4.6. If I is generated by homogeneous polynomials $f_{1}, \ldots, f_{k}$, then $S(I)$ is generated by

$$
L=\left\{\frac{\partial f_{j}}{\partial A_{i}} \mid \text { all } i, j\right\}
$$

Proof. The set $L$ is contained in $S(I)$ by definition. Note that each $f_{j}$ is also in the ideal generated by $L$ by Euler's formula (4.2). Consider a homogeneous 
element $f \in I$. Then there are homogeneous polynomials $h_{1}, \ldots, h_{k}$ such that

$$
f=\sum_{j=1}^{k} h_{j} f_{j}
$$

Differentiating gives

$$
\frac{\partial f}{\partial A_{i}}=\sum_{j=1}^{k}\left(\frac{\partial h_{j}}{\partial A_{i}} f_{j}+h_{j} \frac{\partial f_{j}}{\partial A_{i}}\right)
$$

Using Euler's formula (4.2), both terms on the right are in the ideal generated by $L$. Nonhomogeneous $f \in I$ may be written as the sum of their homogeneous pieces, each in $I$ by the homogeneity of the generators of $I$.

Starting with one generator $f$ in Lemma 4.6 and proceeding by induction gives the following corollary.

Corollary 4.2. For a principal ideal $I=\langle f\rangle$,

$$
\left.S^{(k)}(I)=\left\langle\frac{\partial^{m} f}{\partial A_{1}^{j_{1}} \cdots \partial A_{n}^{j_{n}}}\right| m \leq k, \text { and } \sum_{i=1}^{n} j_{i}=m\right\rangle .
$$

The main result of this section is

Theorem 4.1 (Singular loci are degeneracy loci). Let $W$ be a nonzero configuration of dimension $m$ in $K^{E}$. Then

$$
\operatorname{Sing}_{\geq k} X_{W}=D_{m-k-1}\left(\left.B_{E}\right|_{W}\right)
$$

for $1 \leq k \leq m-1$. In particular,

$$
\operatorname{Mult}_{k} X_{W}=\left.\operatorname{Corank}_{k} B_{E}\right|_{W}
$$

Lemma 4.7 (Singularities determined by restricted configurations). Let $W$ be a nonzero configuration of dimension $m$ in $K^{E}$. For $1 \leq k \leq m-1$,

$$
\operatorname{Sing}_{\geq k} X_{W}=\mathcal{V}\left(\left\langle\Psi_{W^{E-F}}|| F \mid \leq k\right\rangle\right)=\bigcap_{\substack{F \subseteq E \\|F| \leq k}} X_{\left(W^{E-F}, K^{E}\right)}
$$


Proof. Note that when $\Psi_{W}$ is differentiated more than once with respect to the same variable, the derivative is identically zero because no variable occurs more than once in each monomial. Therefore,

$$
S^{(k)}\left(X_{W}\right)=\left\langle\partial_{F} \Psi_{W}|| F \mid \leq k\right\rangle
$$

using Corollary 4.2. By Corollary 4.1 with $H=E-F$,

$$
\left\langle\partial_{F} \Psi_{W}|| F \mid \leq k\right\rangle=\left\langle\Psi_{W^{E-F}}|| F \mid \leq k\right\rangle,
$$

which completes the proof.

Proof of Theorem 4.1. If $B_{E}(a)$ has rank at most $m-k-1$ on $W$, then it has rank at most $m-k-1$ restricted to the subspace $W^{E-F}$. By Lemma 4.3, $\operatorname{dim} W^{E-F}$ is bounded below by $m-k$, so $B_{E}(a)$ is not full rank when restricted to $W^{E-F}$. Therefore, the determinant vanishes:

$$
\left.\operatorname{det} B_{E}(a)\right|_{W^{E-F}}=\Psi_{W^{E-F}}(a)=0 .
$$

If $a \in \mathcal{V}\left(\left\langle\Psi_{W^{E-F}}|| F \mid \leq k\right\rangle\right)$, then $B_{E}(a)$ is degenerate when restricted to all subspaces $W^{E-F}$ for $|F| \leq k$. Note that the subspaces $W^{E-F}$ are intersections of the complete set of hyperplanes $\left\{W^{E-e} \mid e \in E, W \neq W^{E-e}\right\}$. As a result, the conditions of Lemma 2.5 are satisfied, so the rank of $B_{E}(a)$ is at most $m-k-1$.

The statement about multiplicity and corank follows immediately.

Example 4.2 (Trivial configuration). The configuration $W=K^{E}$ has configuration polynomial $\Psi_{W}(A)=\prod_{e \in E} A_{e}$. Its configuration hypersurface is the union of the coordinate hyperplanes. For each $k$ between 1 and $m-1$, Sing ${ }_{\geq k} X_{W}$ is the union of the coordinate linear subspaces of codimension $k+1$. In particular, Sing ${ }_{\geq k}$ has codimension 1 in $\operatorname{Sing}_{\geq k-1}$. For example in $\mathbb{P}\left(K^{3}\right)$, there are three distinct coordinate hyperplanes whose union is $X_{W}$. These hyperplanes meet at $\operatorname{Sing}_{>1} X_{W}$, which consists of the three projective points representing the coordinate axes. The locus $\operatorname{Sing}_{\geq 2} X_{W}=$ $\mathcal{V}\left(A_{1}, A_{2}, A_{3}\right)$ is empty in $\mathbb{P}\left(K^{3}\right)$, though in the affine space $K^{3}$ it is simply the origin.

Remark 4.3. The main theorems in [15] and [12] provide conditions under which degeneracy loci are nonempty. The conditions of these theorems are satisfied for all $k$ between 1 and $m-1$ for $D_{k}\left(\left.B_{E}\right|_{W}\right)$, and hence, $D_{k}\left(\left.B_{E}\right|_{W}\right)=\operatorname{Sing}_{\geq m-k-1} X_{W}$ is nonempty for such $k$. The connectedness 
of these degeneracy loci is a more delicate matter. For example, whether the connectedness theorems in [20] and [14] apply will depend on the specific values of $n, m$, and $k$.

\subsection{Tangent cones}

In this section, I show how to interpret the locus of order at least $k$ singularities in terms of tangent cones. The theory of tangent cones is greatly simplified by restricting the field $K$ to be algebraically closed, so I assume $K$ is algebraically closed for this section.

Let $X$ be a projective hypersurface defined by a homogeneous polynomial $f$. If $a=\left[a_{1}: \cdots: a_{n}\right]$ is a point in $X$ and $a_{i} \neq 0$, then the multivariate Taylor formula in the local coordinates with $a_{i}=1$ expresses $f$ near $a$ as

$$
f\left(\frac{A}{A_{i}}\right)=\left.\sum_{j=0}^{\operatorname{deg} f} \sum_{|J|=j} \frac{1}{J !} \frac{\partial^{J} f}{\partial A^{J}}\right|_{\frac{A}{A_{i}}=\frac{a}{a_{i}}}\left(\frac{A}{A_{i}}-\frac{a}{a_{i}}\right)^{J} .
$$

Therefore, when $a \in \operatorname{Mult}_{k} X$, all the terms with $j<k$ are zero and

$$
f\left(\frac{A}{A_{i}}\right)=\left.\sum_{j=k}^{\operatorname{deg} f} \sum_{|J|=j} \frac{1}{J !} \frac{\partial^{J} f}{\partial A^{J}}\right|_{\frac{A}{A_{i}}=\frac{a}{a_{i}}}\left(\frac{A}{A_{i}}-\frac{a}{a_{i}}\right)^{J} .
$$

Picking out the $k$-th term of Formula (4.3) defines the affine tangent cone

$$
T C_{a} \mathcal{V}(f)=\mathcal{V}\left(\left.\sum_{|J|=k} \frac{1}{J !} \frac{\partial^{J} f}{\partial A^{J}}\right|_{\frac{A}{A_{i}}=\frac{a}{a_{i}}}\left(\frac{A}{A_{i}}-\frac{a}{a_{i}}\right)^{J}\right) .
$$

One may homogenize the leading term at $a$

$$
\begin{aligned}
f_{a, k}\left(\frac{A}{A_{i}}\right) & =\left.\sum_{|J|=k} \frac{1}{J !} \frac{\partial^{J} f}{\partial A^{J}}\right|_{\frac{A}{A_{i}}=\frac{a}{a_{i}}}\left(\frac{A}{A_{i}}-\frac{a}{a_{i}}\right)^{J} \\
& =\left.\frac{1}{a_{i}^{k} A_{i}^{k}} \sum_{|J|=k} \frac{1}{J !} \frac{\partial^{J} f}{\partial A^{J}}\right|_{\frac{A}{A_{i}}=\frac{a}{a_{i}}}\left(a_{i} A-a A_{i}\right)^{J},
\end{aligned}
$$

so the projective tangent cone to $\mathcal{V}(f)$ at $a$ is

$$
\mathbb{T} C_{a} \mathcal{V}(f)=\mathcal{V}\left(\left.\sum_{|J|=k} \frac{1}{J !} \frac{\partial^{J} f}{\partial A^{J}}\right|_{\frac{A}{A_{i}}=\frac{a}{a_{i}}}\left(a_{i} A-a A_{i}\right)^{J}\right) .
$$


The notation $\left(a_{i} A-a A_{i}\right)^{J}$ for a tuple $J=\left(j_{1}, \ldots, j_{n}\right)$ is shorthand for

$$
\left(a_{i} A-a A_{i}\right)^{J}=\prod_{\ell=1}^{n}\left(a_{i} A_{\ell}-a_{\ell} A_{i}\right)^{j_{\ell}}
$$

In particular, if $j_{i}>0$, the product is zero.

Proposition 4.1 (Configuration tangent cone). Let $W$ be a nonzero configuration in $K^{E}$. If $a=\left[a_{1}: \cdots: a_{n}\right]$ is a point of multiplicity $k$ with $a_{i} \neq 0$, then the tangent cone to $X_{W}$ at a is

$$
T C_{a} X_{W}=\mathcal{V}\left(\sum_{\substack{J \subseteq E,|J|=k \\ \operatorname{dim} W^{E-J}=\operatorname{dim} W-k}} C_{J} \Psi_{W^{E-J}}\left(\frac{a}{a_{i}}\right)\left(\frac{A}{A_{i}}-\frac{a}{a_{i}}\right)^{J}\right)
$$

where the $C_{J}$ are nonzero constants independent of a. The projective tangent cone to $X_{W}$ at a is

$$
\mathbb{T} C_{a} X_{W}=\mathcal{V}\left(\sum_{\substack{J \subseteq E,|J|=k \\ \operatorname{dim} W^{E-J}=\operatorname{dim} W-k}} C_{J} \Psi_{W^{E-J}}\left(\frac{a}{a_{i}}\right)\left(a_{i} A-a A_{i}\right)^{J}\right)
$$

If $\Psi_{W}$ is a graph polynomial, all $C_{J}=1$.

Proof. The proposition follows from Equations (4.4) and (4.5) setting $f=$ $\Psi_{W}$. Namely, the $k$-th order term of $\Psi_{W}$ at $a$ is

$$
\Psi_{W, k, a}\left(\frac{A}{A_{i}}\right)=\left.\sum_{|J|=k} \frac{1}{J !} \frac{\partial^{J} \Psi_{W}}{\partial A^{J}}\right|_{\frac{A}{A_{i}}=\frac{a}{a_{i}}}\left(\frac{A}{A_{i}}-\frac{a}{a_{i}}\right)^{J} .
$$

The configuration polynomial has degree at most one in each variable (Proposition 3.1), so only tuples $J$ that are sequences of 0 s and 1 s need to be included in the sum. Such tuples correspond to subsets of $E$. If $|J|=k$, then Lemma 4.5 simplifies $\partial_{J} \Psi_{W}$ to 0 or to $C_{J} \Psi_{W^{E-J}}$ depending on whether $\operatorname{dim} W^{E-J}=\operatorname{dim} W-k$.

The relationship between the rank loci and the singular loci for configuration hypersurfaces is similar to the relationship between the two loci for generic symmetric determinantal loci. I make the relationship explicit in the following Corollary 4.3. For every vector space $W$, the generic symmetric 
degeneracy locus $\mathfrak{X}$ is the locus of points in $\mathbb{P}\left(\mathrm{Sym}^{2} W^{\vee}\right)$ that do not have full rank as a bilinear form on $W$. Suppose that $W$ is a configuration in the based vector space $K^{E}$ so that there is a surjective restriction map

$$
\operatorname{Sym}^{2}\left(K^{E}\right)^{\vee} \stackrel{\pi}{\rightarrow} \operatorname{Sym}^{2} W^{\vee}
$$

Let $Z$ be the kernel of $\pi$. Let $\pi$ also denote the rational map

$$
\mathbb{P}\left(\operatorname{Sym}^{2}\left(K^{E}\right)^{\vee}\right) \rightarrow \mathbb{P}\left(\operatorname{Sym}^{2} W^{\vee}\right)
$$

defined on the complement of $\mathbb{P}(Z)$. Because the fibers of $\pi$ are linear, $\mathbb{P}\left(\operatorname{Sym}^{2}\left(K^{E}\right)^{\vee}\right)$ is a cone over $\mathbb{P}\left(\operatorname{Sym}^{2} W^{\vee}\right)$ with vertex $\mathbb{P}(Z)$.

The family $\mathbb{P}\left(B_{E}(A)\right) \cong \mathbb{P}\left(K^{E}\right)$ is a linear subspace of $\mathbb{P}\left(\operatorname{Sym}^{2}\left(K^{E}\right)^{\vee}\right)$ containing the configuration hypersurface $X_{W}$. Let $L=\mathbb{P}\left(\pi\left(B_{E}(A)\right)\right)$ be the image of the family $\mathbb{P}\left(B_{E}(A)\right)$ in $\mathbb{P}\left(\operatorname{Sym}^{2} W^{\vee}\right)$. Note that $X_{W}$ is a cone over $L \cap \mathfrak{X}$ with vertex $V=\mathbb{P}\left(B_{E}(A)\right) \cap \mathbb{P}(Z)$; that is, $\pi$ is defined on $X_{W}-V$ with image $L \cap \mathfrak{X}$ and linear fibers.

Corollary 4.3 (Intersection of tangent cones). For every $x \in X_{W}-V$,

$$
\operatorname{Mult}_{x} X_{W}=\operatorname{Mult}_{\pi(x)} \mathfrak{X}
$$

and therefore,

$$
\mathbb{T} C_{\pi(x)}(L \cap \mathfrak{X})=L \cap \mathbb{T} C_{\pi(x)} \mathfrak{X},
$$

and similarly for the affine tangent cones. When the vertex $V$ is empty, $\pi$ embeds $\mathbb{P}\left(B_{E}(A)\right)$ into $\mathbb{P}\left(\mathrm{Sym}^{2} W^{\vee}\right)$, and

$$
\mathbb{T} C_{x} X_{W}=L \cap \mathbb{T} C_{x} \mathfrak{X}
$$

Proof. When $\mathfrak{X}$ is cut by the linear section $L$, the leading term at $\pi(x)$ may vanish leading to a higher multiplicity. It is well-known that Mult $_{\pi(x)} \mathfrak{X}$ is the corank of $x$ as a bilinear form on $W$ (see [17] or [13] for example). By Theorem 4.1, Mult $X_{W}$ is also the corank of $x$ as a bilinear form on $W$. Moreover, Mult $X_{W}=$ Mult $_{\pi(x)} L \cap \mathfrak{X}$ because $X_{W}$ is a cone over $L \cap \mathfrak{X}$. In other words, the multiplicity at $\pi(x)$ does not increase when restricting to the linear section $L \cap \mathfrak{X}$. Therefore, the leading term defining $X_{W}$ at $x$ is found by linear substitution of $L$ into the leading term defining $\mathfrak{X}$ at $\pi(x)$, which means that the tangent cone of $L \cap \mathfrak{X}$ is just the linear section of $\mathbb{T} C_{\pi(X)} \mathfrak{X}$. 


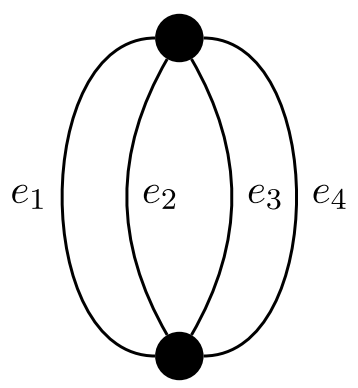

Figure 1: A simple example of a graph.

Example 4.3. Consider the graph $G$ in figure 1. Let $E=\left\{e_{1}, e_{2}, e_{3}, e_{4}\right\}$, and consider the configuration $W=H_{1}(G)$ in $K^{E}$. There are orientations of $G$ for which an integral basis of $H_{1}(G)$ is given by $\left\{\ell_{1}, \ell_{2}, \ell_{3}\right\}$ where

$$
\begin{aligned}
& \ell_{1}=e_{1}+e_{2}, \\
& \ell_{2}=e_{2}+e_{3}, \\
& \ell_{3}=e_{3}+e_{4} .
\end{aligned}
$$

Elements of $\operatorname{Sym}^{2}\left(K^{E}\right)^{\vee}$ and $\operatorname{Sym}^{2} W^{\vee}$ can be represented by symmetric matrices in these bases. Because these matrices are symmetric, I only write the upper triangle to reduce clutter. For $a \in \operatorname{Sym}^{2}\left(K^{E}\right)^{\vee}$ represented by

$$
a=\left(\begin{array}{cccc}
a_{1} & a_{12} & a_{13} & a_{14} \\
* & a_{2} & a_{23} & a_{24} \\
* & * & a_{3} & a_{34} \\
* & * & * & a_{4}
\end{array}\right)
$$

the image of $a$ in $\mathrm{Sym}^{2} W^{\vee}$ can be represented

$$
\pi(a)=\left(\begin{array}{ccc}
a_{1}+2 a_{12}+a_{2} & a_{12}+a_{13}+a_{2}+a_{23} & a_{13}+a_{14}+a_{23}+a_{24} \\
* & a_{2}+2 a_{23}+a_{3} & a_{23}+a_{24}+a_{3}+a_{34} \\
* & * & a_{3}+2 a_{34}+a_{4}
\end{array}\right) .
$$

An element of the family $B_{E}(A)$ is of the form

$$
\left(\begin{array}{cccc}
a_{1} & 0 & 0 & 0 \\
0 & a_{2} & 0 & 0 \\
0 & 0 & a_{3} & 0 \\
0 & 0 & 0 & a_{4}
\end{array}\right)
$$


and its image in $\pi\left(B_{E}(A)\right)$ is

$$
\left(\begin{array}{ccc}
a_{1}+a_{2} & a_{2} & 0 \\
* & a_{2}+a_{3} & a_{3} \\
* & * & a_{3}+a_{4}
\end{array}\right) .
$$

Let a generic element of $\mathrm{Sym}^{2} W^{\vee}$ be denoted

$$
b=\left(\begin{array}{ccc}
b_{1} & b_{12} & b_{13} \\
* & b_{2} & b_{23} \\
* & * & b_{3}
\end{array}\right) .
$$

The image $L=\mathbb{P}\left(\pi\left(B_{E}(A)\right)\right)$ is defined by the ideal

$$
I=\left\langle B_{13}, B_{2}-B_{12}-B_{23}\right\rangle .
$$

The generic symmetric degeneracy locus is defined by the determinant

$$
f(B)=B_{1} B_{2} B_{3}+2 B_{12} B_{23} B_{13}-B_{1} B_{23}^{2}-B_{2} B_{13}^{2}-B_{3} B_{12}^{2} .
$$

The kernel of $\pi$ meets $B_{E}(A)$ at zero, and thus the vertex of the map of projective varieties is empty. In particular, the isomorphism of $\mathbb{P}\left(B_{E}(A)\right)$ with $L$ corresponds to the isomorphism of rings

$$
\begin{aligned}
K[B] / I \stackrel{\pi^{*}}{\longmapsto} K[A] \\
B_{1} \longmapsto A_{1}+A_{2} \\
B_{2} \longmapsto A_{2}+A_{3} \\
B_{3} \longmapsto A_{3}+A_{4} \\
B_{12} \longmapsto A_{2} \\
B_{23} \longmapsto A_{3} .
\end{aligned}
$$

Consider the rank-one matrix

$$
b=\left(\begin{array}{lll}
1 & 0 & 0 \\
0 & 0 & 0 \\
0 & 0 & 0
\end{array}\right)
$$

and note that $b=\pi(a)$ where

$$
a=\left(\begin{array}{llll}
1 & 0 & 0 & 0 \\
0 & 0 & 0 & 0 \\
0 & 0 & 0 & 0 \\
0 & 0 & 0 & 0
\end{array}\right)
$$


Because $b$ has rank one, it must have multiplicity two on both $\mathfrak{X}$ and $X_{W}$. Therefore, all of the first partials of $f$ with respect to the $B$ coordinates vanish, but there are second partials that do not:

$$
\begin{aligned}
\frac{\partial^{2} f}{\partial B_{2} \partial B_{3}}(b) & =b_{1}=1 \\
\frac{\partial^{2} f}{\partial B_{23}^{2}}(b) & =-2 b_{1}=-2 .
\end{aligned}
$$

Therefore, the formula for the tangent cone is

$$
\mathbb{T} C_{b} \mathfrak{X}=\mathcal{V}\left(B_{2} B_{3}-B_{23}^{2}\right) .
$$

Intersecting the tangent cone with the subspace $L$ and transforming to the $A$ coordinates gives

$$
L \cap \mathbb{T} C_{b} \mathfrak{X}=\mathcal{V}\left(\left(A_{2}+A_{3}\right)\left(A_{3}+A_{4}\right)-A_{3}^{2}\right) .
$$

This hyperplane agrees with the computation for $\mathbb{T} C_{a} X_{W}$. Namely, the second partials of $\Psi_{W}$ that do not vanish at $a$ are

$$
\begin{gathered}
\frac{\partial^{2} \Psi_{W}}{\partial A_{2} \partial A_{3}}(a)=a_{1}=1, \\
\frac{\partial^{2} \Psi_{W}}{\partial A_{2} \partial A_{4}}(a)=a_{1}=1, \\
\frac{\partial^{2} \Psi_{W}}{\partial A_{3} \partial A_{4}}(a)=a_{1}=1,
\end{gathered}
$$

which means the tangent cone is defined by

$$
\mathbb{T} C_{a} X_{W}=\mathcal{V}\left(A_{2} A_{3}+A_{2} A_{4}+A_{3} A_{4}\right)
$$

For other linear subspaces $\tilde{L}$, it is possible that there are $b \in \tilde{L} \cap \mathfrak{X}$ such that Mult $_{b} \mathfrak{X} \neq \operatorname{Mult}_{b} \tilde{L} \cap \mathfrak{X}$. For example, consider $\tilde{L}=\left\langle B_{3}, B_{23}\right\rangle$ and

$$
b=\left(\begin{array}{lll}
1 & 0 & 0 \\
0 & 1 & 0 \\
0 & 0 & 0
\end{array}\right)
$$

In this case, the only non-vanishing first partial at $b$ is

$$
\frac{\partial f}{\partial B_{3}}(b)=b_{1} b_{2}-b_{12}=1,
$$


so the tangent cone is

$$
\mathbb{T} C_{b} \mathfrak{X}=\mathcal{V}\left(B_{3}\right)
$$

which contains $\tilde{L}$. In the coordinate ring $K\left[B_{1}, B_{2}, B_{12}, B_{13}\right]$ for $\tilde{L}, \tilde{L} \cap \mathfrak{X}$ is defined by

$$
\bar{f}(B)=-B_{2} B_{13}^{2}
$$

In particular, all first partials of $\bar{f}$ vanish at $b$, and the multiplicity at $b$ has increased by intersecting with $\tilde{L}$.

\section{Conclusion}

I have identified formulas for the derivatives of configuration polynomials as configuration polynomials for restrictions of the original configuration. These formulas allow linear algebra of degenerate bilinear forms to prove my main result relating the multiplicities and ranks of the points in configuration hypersurfaces. The multiplicity-rank relationship is the same as for the generic symmetric determinantal loci, but my approach is quite different. A more geometric approach along the lines of the proof for the generic case would be interesting and may provide a better understanding of the geometry of the incidence variety for the graph hypersurface. Ideally, I would like to use the generic case to prove results upon restricting to configuration hypersurfaces. As these spaces may be highly singular and I have multiplicity information, Stratified Morse Theory seems a promising route to cohomological calculations.

The identification of the second graph polynomial as a configuration polynomial allows the results to apply to both polynomials in the parametric Feynman integral (1.1), but I have only considered momenta with values in a field $K$. In some cases of interest to physicists, non-scalar momenta are required, and I have not pursued that here. The case of quaternionic momenta is handled by Bloch and Kreimer [6]. The configurations defining the first and second graph polynomials come from homology groups relative to zero and one-dimensional subspaces of the momentum $K^{V, 0}$. Allowing higher dimensional subspaces defines higher-order graph polynomials, and it is an open problem to find combinatorial descriptions of them. The review [9] suggests higher analogs to the first two graph polynomials but from a different perspective. Understanding configuration polynomials for $H_{1}(G)$ and $H_{1}(G, p)$ as a version of the all-minor matrix-tree theorem may provide the link between these two versions of higher-order graph polynomials. 


\section{Acknowledgment}

I would like to thank Spencer Bloch for his invaluable guidance during this research and continued encouragement to provide these results to the mathematical community.

\section{References}

[1] P. Aluffi and M. Marcolli, Parametric Feynman integrals and determinant hypersurfaces, (2009), arXiv:0901.2107.

[2] S. Bloch, H. Esnault and D. Kreimer, On motives associated to graph polynomials, Commun. Math. Phys. 267(1) (2006), 181-225.

[3] D. J. Broadhurst and D. Kreimer, Knots and numbers in $\phi^{4}$ theory to 7 loops and beyond, Internat. J. Modern Phys. C6(4) (1995), 519-524.

[4] D. J. Broadhurst and D. Kreimer, Association of multiple zeta values with positive knots via Feynman diagrams up to 9 loops, Phys. Lett. B393(3-4) (1997), 403-412.

[5] S. Bloch and D. Kreimer, Mixed Hodge structures and renormalization in physics, Commun. Num. Theor. Phys. 2(4) (2008), 637-718.

[6] S. Bloch and D. Kreimer, Feynman amplitudes and Landau singularities for 1-loop graphs, (2010), arXiv:1007.0338.

[7] S. Bloch, Transparencies for talk on graph hypersurfaces, IHES Conference on Polylogarithms and Physics, June 2009. http://math. uchicago.edu/ bloch/talk090525.pdf

[8] F. Brown, On the periods of some Feynman integrals, (2009), arXiv:0910.0114.

[9] C. Bogner and S. Weinzierl, Feynman graph polynomials, (2010), arxiv:1002.3458.

[10] D. Doryn, Cohomology of graph hypersurfaces associated to certain Feynman graphs, PhD thesis, University of Duisburg-Essen, September 2008, arXiv:0811.0402.

[11] M. Goresky and R. MacPherson, Stratified Morse theory, Ergebnisse der Mathematik und ihrer Grenzgebiete. 3. Folge, 'A Series of Modern Surveys in Mathematics', no. 14, Springer-Verlag, Berlin; New York, 1988, pp. 147-234. 
[12] W. Graham, Nonemptiness of symmetric degeneracy loci, Amer. J. Math. 127(2) (2005), 261-292.

[13] J. Harris, Algebraic geometry: a first course, Graduate Texts in Mathematics, no. 133, Springer-Verlag, New York, 1992, pp. 295-301.

[14] J. Harris and L. W. Tu, The connectedness of symmetric degeneracy loci: odd ranks, Topics in algebra, Part 2 (Warsaw) (S. Balcerzyk, ed.), Banach Center Publ., 26, PWN, 1990, Appendix to: The connectedness of degeneracy loci, pp. 249-256.

[15] B. Ilic and J. M. Landsberg, On symmetric degeneracy loci, spaces of symmetric matrices of constant rank and dual varieties, Math. Ann. 314(1) (1999), 159-174.

[16] C. Itzykson and J.-B. Zuber, Quantum field theory, McGraw-Hill, New York, 1980.

[17] T. Józefiak, A. Lascoux and P. Pragacz, Classes of determinantal varieties associated with symmetric and skew-symmetric matrices, Math. USSR-Izv. 18(3) (1982), 575-586.

[18] N. Nakanishi, Graph theory and Feynman integrals, Gordon and Breach, New York, 1971.

[19] E. Patterson, Singularities of configuration and graph hypersurfaces, PhD thesis, University of Chicago, June 2009, Freely available from http://disexpress.umi.com/dxweb.

[20] L. W. Tu, The connectedness of symmetric and skew-symmetric degeneracy loci: even ranks, Trans. Amer. Math. Soc. 313(1) (1989), 381-392.

E-mail address: ericpatterson11@gmail.com

Received March 30, 2010 